\title{
Branch-and-bound for bi-objective integer programming *
}

\author{
Sophie N. Parragh \\ Fabien Tricoire \\ Institute of Production and Logistics Management \\ Johannes Kepler University, Linz, Austria \\ \{sophie.parragh,fabien.tricoire\}@univie.ac.at
}

September 19, 2018

\begin{abstract}
In bi-objective integer optimization the optimal result corresponds to a set of non-dominated solutions. We propose a generic bi-objective branch-and-bound algorithm that uses a problem-independent branching rule exploiting available integer solutions and takes advantage of integer objective coefficients. The developed algorithm is applied to bi-objective facility location problems, to the bi-objective set covering problem, as well as to the bi-objective team orienteering problem with time windows. In the latter case, lower bound sets are computed by means of column generation. Comparison to state-of-the-art exact algorithms shows the effectiveness of the proposed branch-and-bound algorithm.
\end{abstract}

\section{Introduction}

We live in a world full of trade-offs. In almost every problem situation we encounter, it is difficult to define the one and only goal to aim for, especially whenever more than one decision maker or stakeholder is involved. Thus, many if not all practical problems involve several and often conflicting objectives. Prominent examples are profitability or cost versus environmental concerns (Ramos et al. 2014) or customer satisfaction (Braekers et al. 2016). Whenever it is not possible to aggregate these conflicting objectives in a meaningful way, it is recommendable to produce the set of trade-off solutions in order to elucidate their trade-off relationship.

In this paper, we propose a branch-and-bound framework which produces the optimal set of trade-off solutions for optimization problems with two objectives which can be modeled as integer linear programs. The main contributions are a new problem-independent branching rule for bi-objective optimization, building up on the Pareto branching idea of Stidsen et al. (2014), and several improvements exploiting the integrality of objective function values. We compare our framework to existing general purpose state-of-the-art frameworks, such as the $\epsilon$-constraint framework and the balanced box method, and to results obtained by means of another branch-and-bound algorithm developed by Belotti et al. (2013). Furthermore, we apply it to a bi-objective orienteering problem for which lower bound sets are produced using column generation. Although column generation has been used in the context of bi-objective optimization, e.g. in the context of robust airline crew scheduling (Tam et al. 2011), vehicle routing (Sarpong et al.|2013), and bi-objective linear programming (Raith et al. 2012), this is, to the best of our knowledge, the first time column generation is used in a bi-objective branch-and-bound algorithm, resulting in a bi-objective branch-and-price algorithm.

The paper is organized as follows. In Sections 2 and 3 , we give preliminaries and we review related work. In Section 4, we present the key ingredients to our branch-and-bound framework and in Section 5 we discuss the proposed enhancements. Experimental results and how we embed column generation into our branch-and-bound framework are presented in Section 6 . Section 7 concludes the paper.

${ }^{*}$ Accepted for publication in INFORMS Journal On Computing; doi: 10.1287/ijoc.2018.0856 


\section{Preliminaries}

The aim of this paper is to solve bi-objective integer linear programs of the following form:

$$
\begin{array}{cl}
\min _{x} & f(x)=\left(f_{1}(x), f_{2}(x)\right) \\
\text { s.t. } & A x=b \\
& x_{i} \quad \text { integer }
\end{array}
$$

where $f(x)$ is the vector of objective functions, $A$ is the constraint matrix of size $m \times n, b$ the right hand side vector of size $m$, and $x_{i}$ the decision variables which are restricted to integer values. An important subclass are binary (combinatorial) problems.

In formulation (1) we strive for identifying the set of Pareto optimal solutions according to the given objectives. A feasible solution is Pareto optimal if there does not exist any feasible solution which dominates it, i.e. which is better in one objective and not worse in any other objective. The image of a Pareto optimal solution in criterion space is referred to as non-dominated point. Each non-dominated point may represent more than one Pareto optimal solution. We aim at generating all non-dominated points, i.e. at least one Pareto optimal or efficient solution per non-dominated point.

Depending on the structure of the objective functions and the feasible domains of the variables, the set of all non-dominated points may take different shapes. In the case where all variables assume continuous values, it corresponds to the boundary of the convex hull of the image of all feasible solutions in criterion space. It can be characterized by the extreme points of the boundary. These extreme points can be computed efficiently, e.g. by the weighted sum method of Aneja and Nair (1979). It combines the two objectives into a single one, resulting in $\left(w_{1} f_{1}(x)+w_{2} f_{2}(x)\right)$, with $w_{1}$ and $w_{2}$ giving the weights of objective one and two, respectively. These weights are changed systematically so as to obtain the entire set of extreme points. In the case where all variables may only take integer values, which we look at, the set of non-dominated points is composed of points which may or may not lie on the boundary of the convex hull in criterion space. Solutions whose images in criterion space correspond to extreme points are called extreme (supported) efficient solutions, those that correspond to points that lie on the boundary are called supported efficient solutions and those that correspond to points that lie in the interior of the convex hull but are still non-dominated are called non-supported efficient solutions. In the integer case, the set of extreme points can also be computed by means of the weighted sum method.

Additional important notions in multi-objective optimization are ideal point and nadir point. The ideal point is constructed by combining the best possible values for each objective function. It dominates all non-dominated points. The nadir point is its opposite, a point that is dominated by all non-dominated points. It is constructed by combining the worst possible values for each objective function of all efficient solutions. Both notions have been used in the context of branch-and-bound algorithms for multi-objective optimization. For a more detailed introduction to multicriteria decision making, we refer to Ehrgott (2005).

\section{Related work}

Exact approaches in multi-objective (mixed) integer programming can be divided into two classes: those that work in the space of objective function values (referred to as criterion space search methods, e.g. by Boland et al. (2015a)) and those that work in the space of decision variables (generalizations of branch-and-bound algorithms).

Criterion space search methods solve a succession of single-objective problems in order to compute the set of Pareto optimal solutions. Therefore, they exploit the power of state-of-the-art mixed integer programming solvers. This appears to be one of their main advantages in comparison to generalizations of branch-andbound algorithms (Boland et al. 2015b). However, many combinatorial single-objective problems cannot be solved efficiently by commercial solvers, e.g. the most efficient exact algorithms in the field of vehicle routing rely on column generation based techniques (see e.g. Baldacci et al. 2012). Thus, especially in this context but also in general, it is not clear whether a criterion space search method or a bi-objective branch-and-bound algorithm is more efficient. 
One of the most popular criterion space search methods is the $\epsilon$-constraint method, first introduced by Haimes et al. (1971). It consists in iteratively solving single-objective versions of a bi-objective problem. In every step, the first objective is optimized, but a constraint is updated in order to improve the quality of the solution with regards to the second objective. Thus all non-dominated points are enumerated. The $\epsilon$-constraint method is generic and simple to implement and it is among the best performing criterion space search algorithms when applied, e.g. to the bi-objective prize-collecting Steiner tree problem (Leitner et al. 2014).

Recently, the balanced box method for bi-objective integer programs has been introduced by Boland et al. (2015a). Optimal solutions are computed for each objective and they define a rectangle. This rectangle is then split in half and in each half, the objective which has not been optimized yet in this half of the rectangle is then optimized. At this stage there are two non-overlapping rectangles. The same procedure is repeated recursively on these rectangles.

Generalizations of branch-and-bound to multiple objectives for mixed 0-1 integer programs are provided by Mavrotas and Diakoulaki (1998). Their bounding procedure considers an ideal point for the upper bound (they work on a maximization problem), consisting of the best possible value for each objective at this node, and keeps branching until this ideal point is dominated by the lower bound set. Vincent et al. (2013) improve the algorithm by Mavrotas and Diakoulaki (1998), most notably by comparing bound sets instead of ideal points in the bounding procedure. Masin and Bukchin (2008) propose a branch-and-bound method that uses a surrogate objective function returning a single numerical value. This value can be treated like a lower bound in the context of single objective minimization and conveys information on whether the node can be fathomed or not. They illustrate their method using a three-objective scheduling problem example but no computational study is provided. Sourd and Spanjaard (2008) use separating hyperplanes between upper and lower bound sets in order to discard nodes in a general branch-and-bound framework for integer programs. The concept is in fact similar to the lower bound sets defined by Ehrgott and Gandibleux (2006). Jozefowiez et al. (2012) introduce a branch-and-cut algorithm for integer programs in which discrete sets are used for lower bounds, so nodes can be pruned if every point in the lower bound set is dominated by a point in the upper bound set. Partial pruning is used in order to discard parts of the solutions represented by a given node of the search tree, thus speeding up the whole tree search. Belotti et al. (2013) are the first to introduce a general-purpose branch-and-bound algorithm for bi-objective mixed integer linear programming, where the continuous variables may appear in both objective functions. They build up on the previous work by Visée et al. (1998), Mavrotas and Diakoulaki (1998), Sourd and Spanjaard (2008) and Vincent et al. (2013) and like them, they use a binary branching scheme. Improved fathoming rules are introduced in order to discard more nodes during tree search. Adelgren et al. (2014) propose an efficient data structure to store and update upper bound sets in the context of mixed integer programming and illustrate its efficiency using the algorithms of Belotti et al. (2013) and of Adelgren and Gupte (2016).

Ideas most related to ours are presented by Stidsen et al. (2014). They provide a branch-and-bound algorithm to deal with a certain class of bi-objective mixed integer linear programs. They propose two binary branching schemes that exploit partial dominance of already visited integer solutions and the current upper bound set, respectively. Our approach relies on bound sets to represent the lower bound, in contrast to Stidsen et al. (2014) who solve one scalarized single objective problem in each node of their branch-andbound tree. In addition, in our scheme, the number of child nodes depends on the number of currently non-dominated portions which are obtained after applying what we call filtering of the lower bound set by the current upper bound set. Ideas similar to the Pareto branching concept of Stidsen et al. (2014) and its extension to bound sets presented in this paper, which we name objective space branching, are also being explored by Adelgren and Gupte (2016) in the context of a branch-and-bound method for multi-objective mixed integer programming and by Gadegaard et al. (2016a) in the context of a bi-objective branch-and-cut algorithm.

Furthermore, we also propose several improvements exploiting the integrality of objective functions. These improvements, which we call segment tightening, lower bound lifting, and integer dominance allow to disregard even larger regions of the objective space. We illustrate their impact on the overall performance of the algorithm in the experimental results section. 


\section{A branch-and-bound framework for bi-objective integer opti- mization}

Branch-and-bound is a general purpose tree search method to solve (mixed) integer linear programs. As its name suggests, it has two main ingredients: branching and bounding. Branching refers to the way the search space is partitioned, while bounding refers to the process of using valid bounds to discard subsets of the search space without compromising optimality. In the following, we first describe the general framework of our bi-objective branch-and-bound (BIOBAB). We then describe its different ingredients in further detail.

\subsection{General idea}

Our BIOBAB is similar to a traditional single-objective branch-and-bound algorithm, except for the fact that we compare bound sets, instead of single numerical values.

In the beginning the upper bound (UB) set is empty. It is updated every time an integer solution $x$ is obtained. One of three things can happen:

1. if $x$ is dominated by at least one solution from the UB set, then $x$ is discarded,

2. if some solutions from the UB set are dominated by $x$, then these solutions are discarded and $x$ is added to the UB set,

3. if $x$ neither dominates nor is dominated by any solution from the UB set, $x$ is directly added to the UB set.

These conditions can be tested in linear time. If the UB set is sorted according to the values of one objective, it is possible to check dominance in logarithmic time using binary search.

In each branch-and-bound node, lower bound (LB) sets are computed by means of an algorithm similar to the weighted sum method of Aneja and Nair (1979), described in detail in Section 5.2, with either the linear relaxation or the original integer program (IP), since both yield valid bound sets (Ehrgott and Gandibleux 2006).

A branch-and-bound node contains information on branching decisions as usual, but also a bound for each objective. This is used to partition the objective space. The objective bounds at the root node can be known valid bounds, or infinite values.

Once the LB set at a given node has been calculated, we apply what we call filtering (see Section 4.3): the current UB set and the current LB set are compared and a set of non-dominated portions of the current LB set are returned. In the case where this set is empty, the node can be fathomed. Otherwise branching may be required; the filtered LB set is then used as input for the branching procedure (see Section 4.4). If the LB set corresponds to a leaf (i.e. the node corresponds to a unique integer feasible solution), or if it is completely dominated following filtering, then the node can be fathomed and branching is not necessary. Otherwise a number of child nodes are generated, which are added to the list of branch-and-bound nodes to be processed. Once all nodes have been explored, the search is over and the complete Pareto set for the original problem is contained in the UB set.

We now present the concept of lower bound segment which is used in the bounding, filtering and branching procedures and which is also key to the proposed improvements discussed in Section 5.

\subsection{Lower bound segments}

In the following we assume that a LB set has been produced. As mentioned above, it is described by the extreme points of the convex hull (of the image of the feasible set in objective space) for the linear relaxation of the problem (or the original IP).

To any two consecutive extreme points $p=\left(p_{1}, p_{2}\right)$ and $q=\left(q_{1}, q_{2}\right)$ we can associate the subset of objective space that is covered or dominated and that represents the possibility to find feasible solutions to the integer problem. To define this covered space, we associate to each pair of extreme points a top-right 


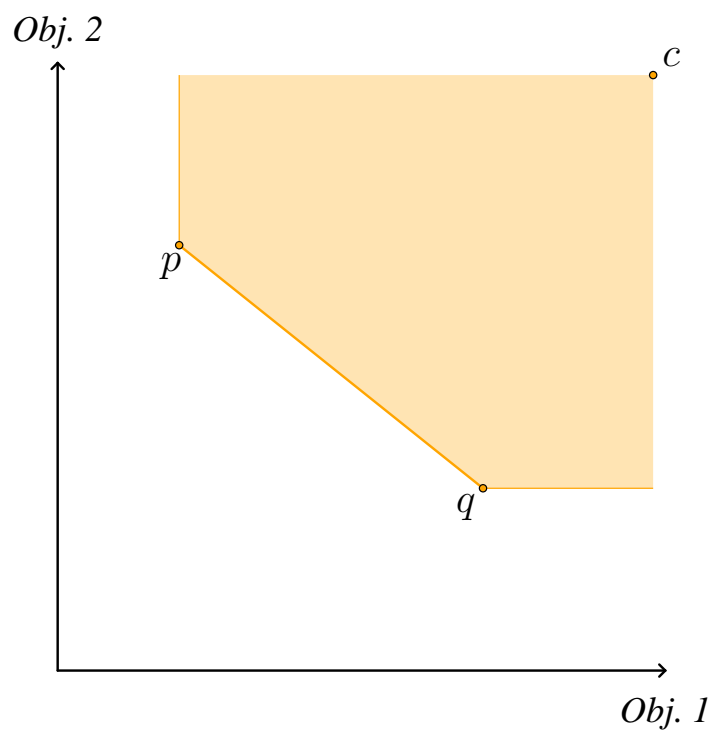

Figure 1: Lower bound segment defined by the two extreme points $p$ and $q$, and local nadir point $c$.

corner (or local nadir) point $c=\left(c_{1}, c_{2}\right)$ of which coordinates are valid single-objective upper bounds, one per objective, on the space dominated by the two extreme points and the line connecting them.

Let $\Xi$ be the set of all points in the objective space which are associated to feasible solutions to the bi-objective integer problem at hand. We now formally introduce the concept of lower bound segment that we use to represent LB sets.

Definition 1 Three points $p=\left(p_{1}, p_{2}\right), q=\left(q_{1}, q_{2}\right)$, and $c=\left(c_{1}, c_{2}\right)$, such that $p_{1}<q_{1} \wedge p_{2}>q_{2}$, define a lower bound segment $s$ iff $\left\{\left(z_{1}, z_{2}\right) \in \Xi \mid z_{2}<a z_{1}+b\right\}=\emptyset$, where $a=\left(q_{2}-p_{2}\right) /\left(q_{1}-p_{1}\right)$ is the slope of the line defined by the two points and $b=p_{2}-a \cdot p_{1}$ is the $y$-intercept of this same line; $c$ is a valid local nadir point, such that $c_{1} \geq q_{1}$ and $c_{2} \geq p_{2}$.

Thus, any point $z=\left(z_{1}, z_{2}\right) \in \Xi$ is on or above the line defined by $p$ and $q$. Note that we do not include the case where $p=q$ into our definition. For convenience, we extend the definition of segments to allow equal points:

Definition 2 Two points $p=\left(p_{1}, p_{2}\right)$ and $c=\left(c_{1}, c_{2}\right)$ define a lower bound segment $s$ iff there does not exist a feasible point $z$ that dominates $p ; c$ is a valid local nadir point, such that $c_{1} \geq p_{1}$ and $c_{2} \geq p_{2}$

Figure 1 depicts such a lower bound segment (shaded). In the following we use object-oriented notation to refer to the attributes defining a given segment: if extreme points $u, v$ and local nadir point $w$ define a segment $s$, then, $s . p=u, s . q=v$ and $s . c=w$. Additionally, $s . a$ is the slope of the line going through $s . p$ and $s . q$, while $s . b$ is its $y$-intercept. Graphically, a segment $s$ is a convex polygon defined by the points s.p, $s . q,\left(s . c_{1}, s . q_{2}\right), s . c,\left(s . p_{1}, s . c_{2}\right)$.

In the most basic case the coordinates of the local nadir point can be arbitrarily large values. If the two extreme points of the set of non-dominated points are known, we can deduce a tighter valid local nadir point from them. Similarly, we can associate to any LB set a valid local nadir point by considering, for each objective, the maximum value among all local nadir points.

Here we note that we can partition the objective space, using values for any of the two objectives, or any linear combination of both with positive weights (as is done in Stidsen et al. 2014). In our BIOBAB, we partition the objective space using different intervals for the first objective to split the LB set into vertical stripes. 


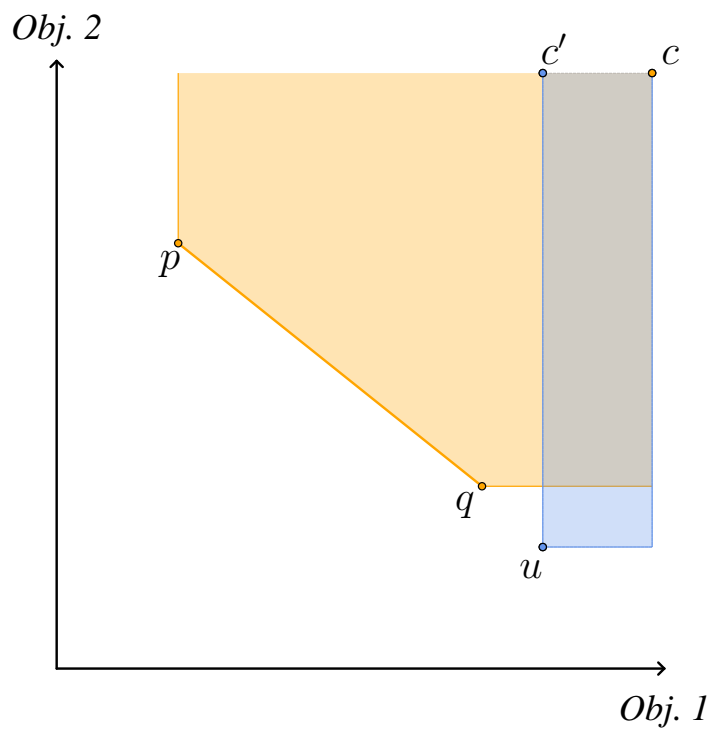

Figure 2: Space dominated by the segment between points $p$ and $q$ can be reduced by removing from it the space dominated by upper bound point $u$. Therefore local nadir point $c$ can be improved to $c^{\prime}$

In practice, this allows us to improve the initial local nadir point for segments of a connected sequence of segments: for any two segments connected by point $u=\left(u_{1}, u_{2}\right)$, we can cut from the space covered by the left segment all points $z=\left(z_{1}, z_{2}\right)$ such that $z_{1} \geq u_{1}$, since these points are also covered by the right segment. By doing so, we partition the objective space covered by the LB set into different LB segments with associated local nadir points.

UB sets can also be used to provide better local nadir points. For instance, following our previous example from Figure 1, consider point $u$, being the image of a feasible solution in objective space, such that $u_{1} \geq q_{1}$ and $u_{2}<q_{2}$, then $u_{1}$ defines a valid bound on the first objective of the nadir point, since any point $v$ such that $v_{1} \geq u_{1}$ and $v_{2} \geq q_{2}$ is dominated by $u$. This example is described graphically in Figure 2 for the depicted segment, using UB point $u$, the initial local nadir point $c$ can be improved to $c^{\prime}$.

The fact that LB sets can be reduced using UB sets is the basis for a bi-objective branching rule that is presented in Section 4.4 .

\subsection{Bound set filtering and node fathoming}

One major difficulty in previous bi-objective branch-and-bound approaches lies in the evaluation of the dominance of a given LB set by a given UB set. Multiple fathoming rules have been developed over the years (see Belotti et al. 2016, for the current state of the art). We now introduce the fathoming rule used in BIOBAB.

As seen above, it is possible to split a LB set into LB segments (with associated local nadir points). If we establish that each segment of a current LB set is dominated by the UB set, then we also establish that the whole LB set is dominated, therefore the current node of the branch-and-bound tree can be fathomed. In order to determine if a LB segment is dominated by an UB set, we simply subtract the space dominated by the UB set from the LB segment: if the remaining space is empty then the LB segment is dominated, otherwise this segment may be tightened (as shown for example in Figure 2).

Algorithm 1 details how to subtract the space covered by an UB point $u=\left(u_{1}, u_{2}\right)$ from a LB segment $s$. It shares some similarities with the dominance rules used by Vincent et al. (2013), but considers more cases. Notably, it updates the local nadir point even in cases where $u$ does not dominate any point on the line connecting s.p and s.q. We use the object-oriented notation defined above. A pair $\left(z_{1}, z_{2}\right)$ represents a point 
and $\operatorname{Segment}(p, q, c)$ is used to create a segment out of points $p, q$ and $c$. Lines 2-7 deal with the case where $u$ does not dominate any point on the line connecting s.p and s.q. In that case, $u$ may still be above s.p or to the right of $s . q$, in which cases there is potential for improving s.c. If $u$ is to the right of $s . q$ and below $s . q$ (Lines 5-7), we are in the situation depicted in Figure 2 and $s . c_{1}$ can be reduced to $\min \left(s . c_{1}, u_{1}\right)$. Similarly, if $u$ is above $s . p$ and to the left of $s . p$ (Lines 3-4), s.c $c_{2}$ can then be reduced to $\min \left(s . c_{2}, u_{2}\right)$. Lines 8-15 deal with all the cases when at least one part of the line connecting $s . p$ and $s . q$ is dominated by $u$. There are four different cases: (i) $u$ does not dominate s.p (Lines 9-11), (ii) $u$ does not dominate s.q (Lines 12-14), (iii) $u$ dominates neither s.p nor s.q, resulting in two new segments (both clauses in Lines 9-11 and 12-14 are matched), and (iv) $u$ dominates both s.p and s.q. In case (iv) no clause is matched in the algorithm, therefore an empty set is returned.

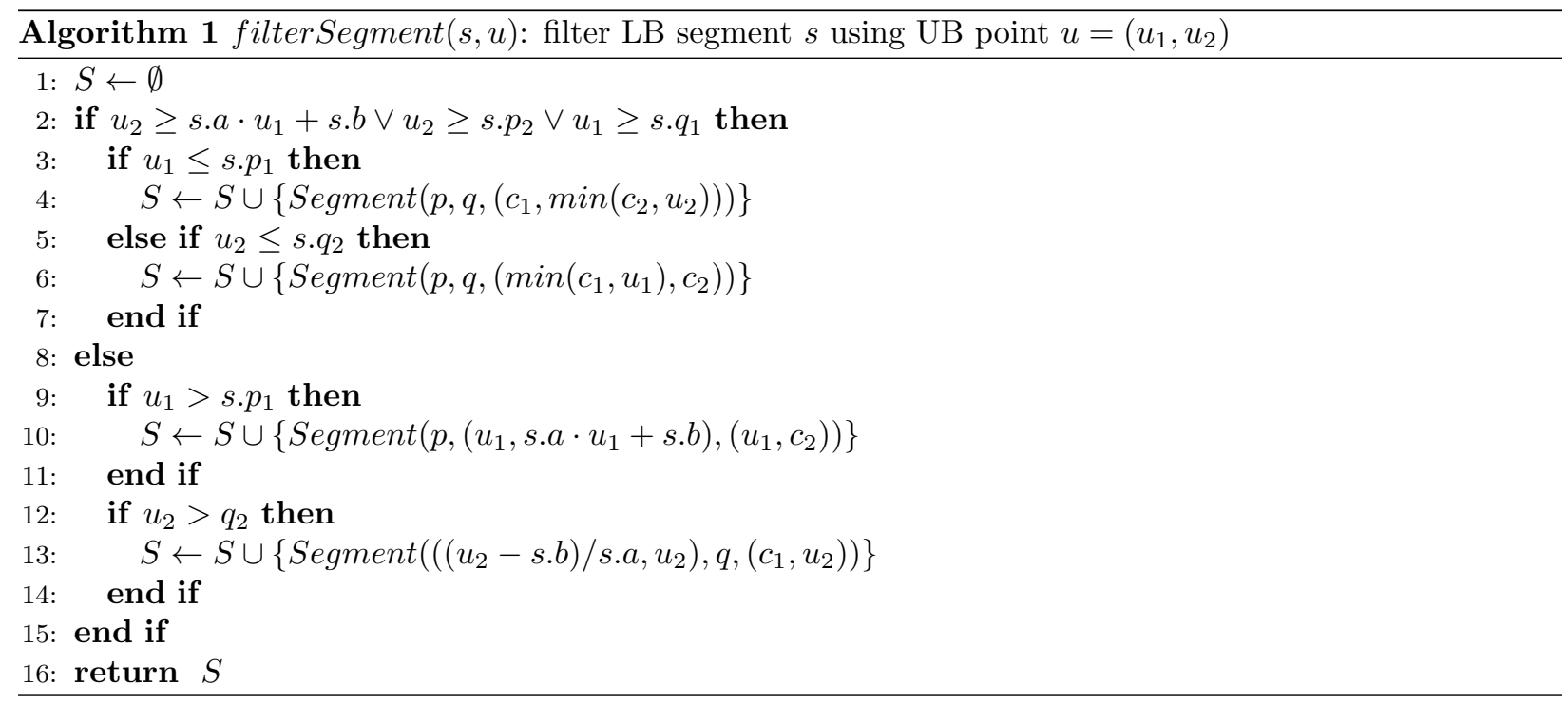

Now in order to filter a whole LB set using an UB set, we filter each segment in the LB set with each point in the UB set. If the remaining LB set is empty then the node can be fathomed, otherwise branching is required.

\subsection{Branching procedure}

When dealing with bi-objective bound sets, a major challenge is that the LB set at a given node of the branchand-bound tree may be partially dominated but not completely, therefore the node cannot be fathomed. This is illustrated in Figure 3 .

In order to disregard those portions of the objective space which are dominated by the UB set, we use the following branching rule which we name objective space branching: each contiguous subset of the filtered LB set gives rise to a new branch and the objective space between those parts is simply ignored. To generate the branch for a given subset, we consider its local nadir point $c=\left(c_{1}, c_{2}\right)$ and add two cutting planes so that both objectives are better than the value at this local nadir point: $f_{1}(x) \leq c_{1}$ and $f_{2}(x) \leq c_{2}$. In practice, this is achieved by setting the objective bounds of the corresponding child nodes using $c$.

Branching on objective space has no impact on decision space, i.e. computing lower bounds for these child nodes will generate the same LB set, albeit in several pieces, one per branch in objective space. Therefore, objective space branching (OSB) never happens alone; branching on decision space is systematically also performed at the same time. The end result is that each node in the search tree contains at least one new branching decision on decision space, and optionally a branching decision on objective space.

In general, decision-space branching is problem specific. However, we can still make the general remark that there is no reason to systematically use the same variable for decision-space branching in every OSB child node: each OSB child has its own separate LB set, and there is no reason to branch on the same 


\section{Obj. 2}

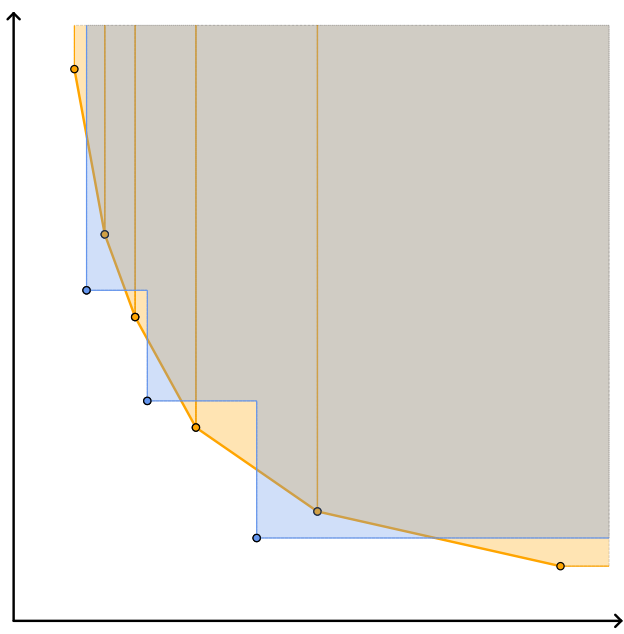

Obj. 2

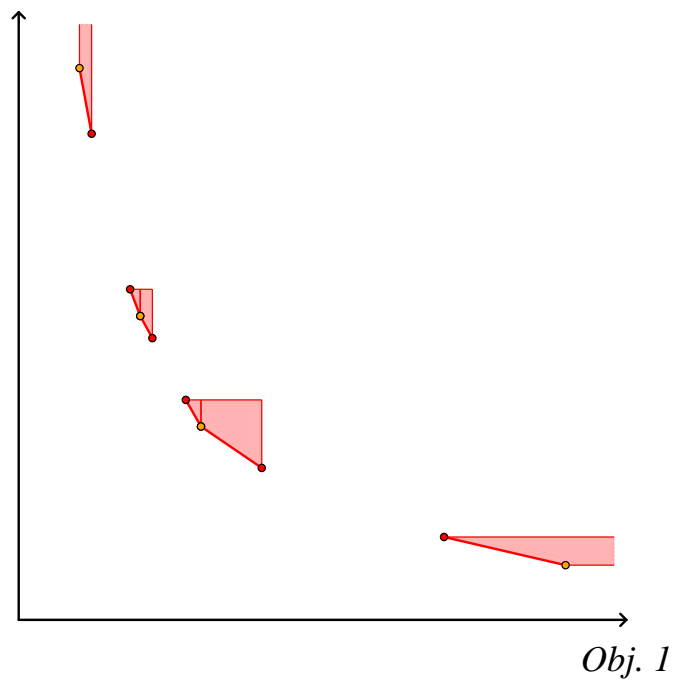

Figure 3: LB set is partially dominated by UB set, the node cannot be fathomed.

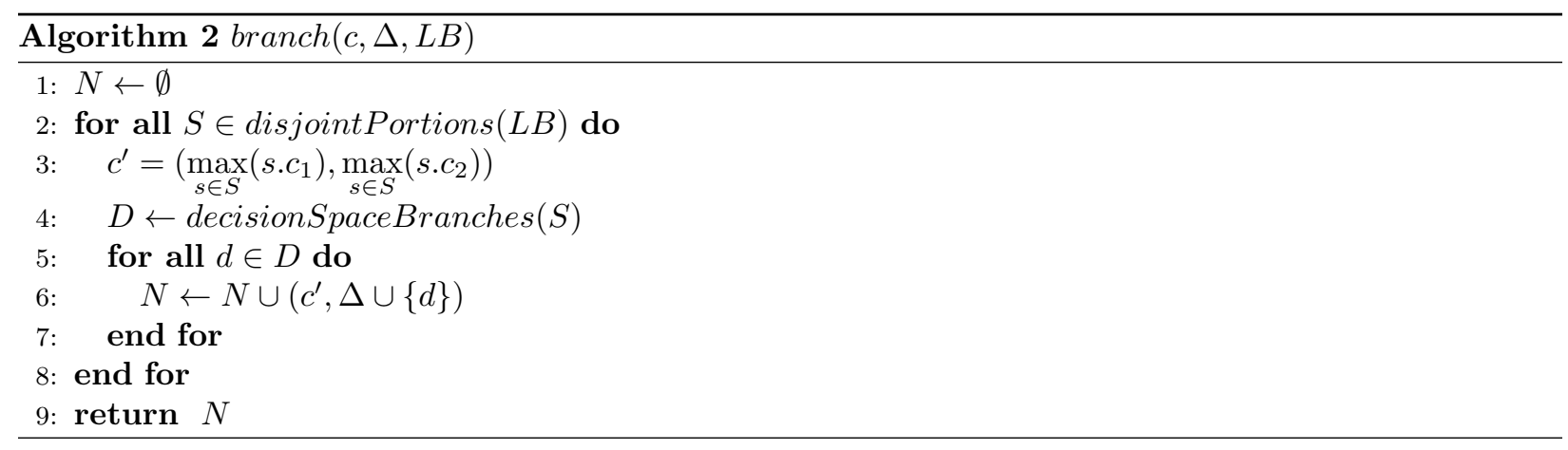

decision variables when considering different disjoint LB sets. For this reason, OSB is always applied first in order to reduce the search space; for each OSB child and associated LB set, decision-space branching is conducted independently. The end result might still be that each OSB child branches on the same variable, depending on the branching rule.

Algorithm 2 gives the branch function of our branch-and-bound framework. It takes a node of the branch-and-bound tree and an LB set as input and returns a set of nodes. A node is represented as a pair $(c, \Delta)$ where $c$ represents the objective bounds (local nadir point) for this node and $\Delta$ is the set of branching decisions for this node.

In the example of Figure 3 , function branch considers four disjoint regions, each of them generating their own set of branching decisions.

We note here that even if an IP is used to compute the LB set, any given variable may take different values over a same LB set; therefore branching on decision space is still necessary.

\section{Improvements based on the integrality of objective functions}

When solving integer programs, in many cases, objective values of feasible integer solutions only take integer values. For pure integer programs, it is in practice almost always possible to use integer coefficients. The 
Obj. 2

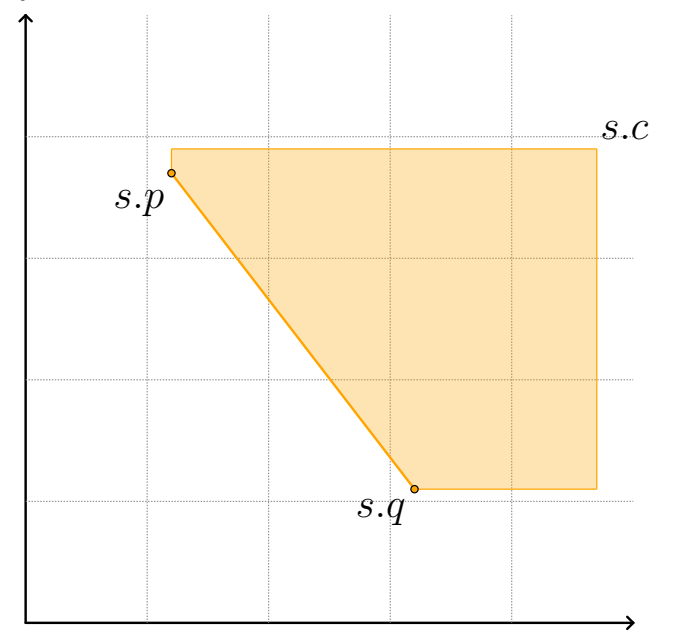

Obj. 1
Obj. 2

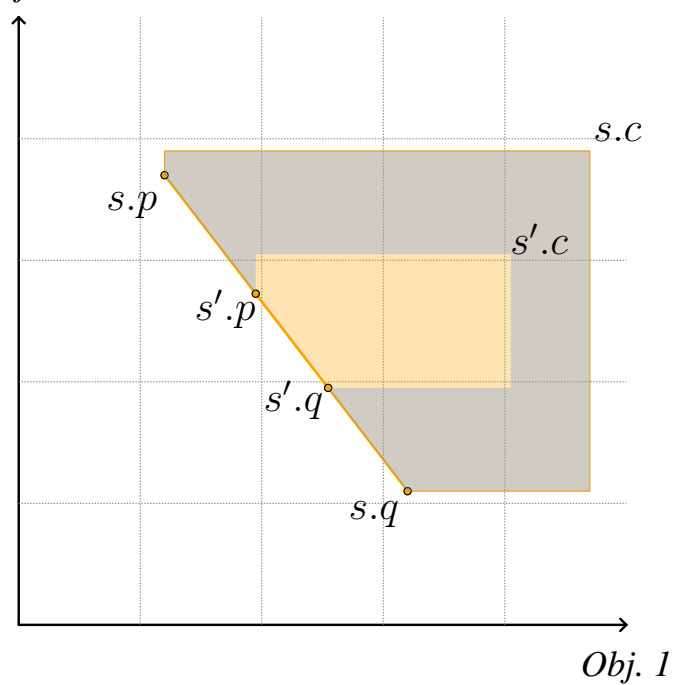

Figure 4: The space covered by a LB segment contains contiguous regions which do not contain any point with integer coordinates; these parts are irrelevant to the search. Tightened segment $s^{\prime}$ contains all the integer solutions contained by original segment $s$.

reasons include the fact that LP solvers have precision limitations, and that time-efficient floating-point numbers representations also have precision limitations. So rounding floating-point numbers is almost always inevitable, and if numbers are rounded to $d$ decimals then they may as well be multiplied by $10^{d}$ and considered integers. We note that this property is exploited by other methods as well. For instance, the $\epsilon$-constraint framework uses a known $\epsilon$ value which in our case would be 1 . The balanced box method also relies on similar $\epsilon$ values. In some cases, it is even possible to use values higher than 1 , as long as these values are valid: for instance if every coefficient for a given objective function is integer, then the greatest common divisor of these coefficients can be used as a valid value. For the sake of simplicity and without loss of generality, we consider in the following that this valid value is 1 . We now explore possibilities to enhance our bi-objective branch-and-bound by exploiting the fact that objective values of feasible solutions are always integer.

Any given LB segment covers a part of the objective space, but also includes subsets not containing any point with integer coordinates. Such subsets can be disregarded during the search. This is illustrated in Figure 4, where dashed lines represent integer values for each objective. This general idea can be exploited in several ways in order to speed up the search.

\section{$5.1 \quad$ Segment tightening}

First, any LB segment which does not cover any integer vector can be discarded. This can be tested in $\mathcal{O}(1)$.

We first investigate the case where $s . p \neq s . q$, then $s . a$ and $s . b$ are defined:

Proposition 1 Segment $s$ with $s . p \neq s . q$ covers integer vectors iff $\left\lfloor s . c_{2}\right\rfloor \geq s . a\left\lfloor s . c_{1}\right\rfloor+s . b \wedge\left\lfloor s . c_{2}\right\rfloor \geq$ $s . q_{2} \wedge\left\lfloor s . c_{1}\right\rfloor \geq s . p_{1}$.

Proof 1 A segment $s$ is a polygon defined by the points s.p, s.q, $\left(s . c_{1}, s . q_{2}\right)$, s.c, $\left(s . p_{1}, s . c_{2}\right)$. The coordinates of any integer point $z=\left(z_{1}, z_{2}\right)$ within this polygon have to satisfy $s . p_{1} \leq z_{1} \leq s . c_{1}$ and $s . q_{2} \leq z_{2} \leq s . c_{2}$ and $z_{2} \geq s . a \cdot z_{1}+$ s.b. The integer point closest to the local nadir point s.c which has the potential to lie within the polygon has coordinates $\left(\left\lfloor s . c_{1}\right\rfloor,\left\lfloor s . c_{2}\right\rfloor\right)$. Any other integer point $z=\left(z_{1}, z_{2}\right)$ contained within the region 


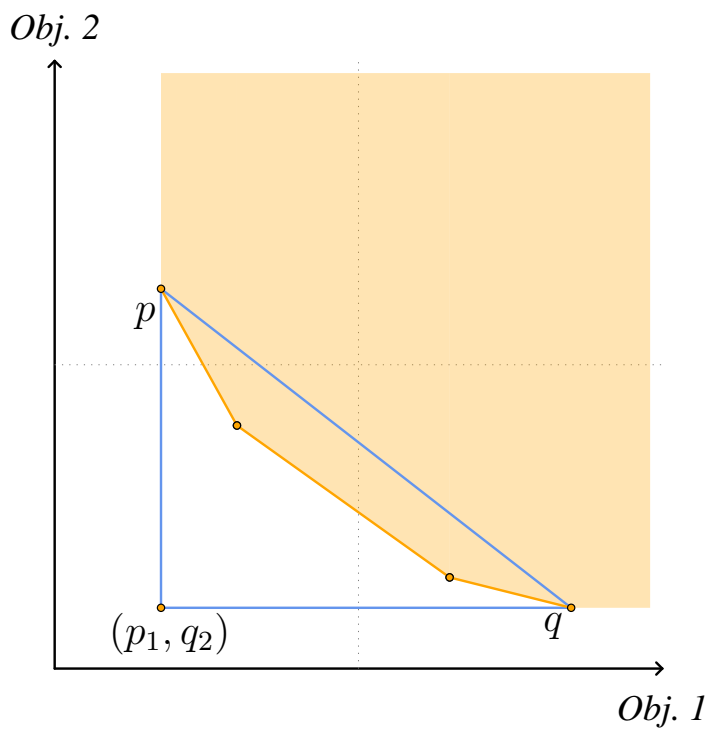

Figure 5: Segment between points $p=\left(p_{1}, p_{2}\right)$ and $q=\left(q_{1}, q_{2}\right)$ defines a valid LB segment because there is no vector of integer coordinates in the triangle defined by $p, q$ and $\left(p_{1}, q_{2}\right)$.

of the segment must have coordinates $z_{1} \leq\left\lfloor s . c_{1}\right\rfloor$ and $z_{2} \leq\left\lfloor s . c_{2}\right\rfloor$. Therefore, if $\left(\left\lfloor s . c_{1}\right\rfloor,\left\lfloor s . c_{2}\right\rfloor\right)$ is contained within $s, s$ covers at least one integer point.

The special case of segments defined by two points only (see Definition 2 is even simpler, as the space covered by such a segment is a rectangle. Consider the segment defined by point $p=\left(p_{1}, p_{2}\right)$ and local nadir point $c=\left(c_{1}, c_{2}\right)$, then this segment contains integer points iff $\left\lfloor c_{1}\right\rfloor \geq z_{1} \wedge\left\lfloor c_{2}\right\rfloor \geq z_{2}$.

This is tested right after filtering: only the segments in the LB set that cover an integer point are kept. This enhancement is called segment tightening.

\subsection{Lower bound lifting}

Second, we can use the fact that feasible integer solutions have integer objective values in order to speed up our LB set computation procedure. We call this procedure bound (it is given in Algorithm 3).

As in the original algorithm of Aneja and Nair (1979), procedure bound successively computes supported points, starting with the two points at the two ends of the efficient frontier, using lexicographic minimum objective functions $\operatorname{lexmin}\left(f_{1}, f_{2}\right)$ and $\operatorname{lexmin}\left(f_{2}, f_{1}\right)$. This is similar to what is done by Boland et al. (2015a) in the context of the balanced box method.

For any two given points, solving a certain weighted-sum problem determines whether there exists another supported solution "between" these two. However, it can happen that these two points already describe a valid LB segment (given an appropriate nadir point), regardless of any other extreme supported point between them. If there is no point with integer coordinates in the triangle defined by $p=\left(p_{1}, p_{2}\right), q=\left(q_{1}, q_{2}\right)$ and $\left(p_{1}, q_{2}\right)$, then $p$ and $q$ already define a valid LB segment and there is no need to investigate these two points further. We note that point $\left(p_{1}, q_{2}\right)$ can also be disregarded because it dominates both $p$ and $q$, although it is already established that they are both non-dominated. Moreover, if the line connecting $p$ and $q$ is not a part of the convex hull boundary then it defines a tighter lower bound than the one induced by the actual convex hull boundary between $p$ and $q$. This situation is illustrated in Figure 5 , where $p$ and $q$ define a valid lower bound segment (given an appropriate local nadir point).

As previously, testing for the existence of an integer vector in the given triangle can be achieved in $\mathcal{O}(1)$ and sometimes allows to skip some stages of the algorithm while providing a tighter LB set. In the notation 


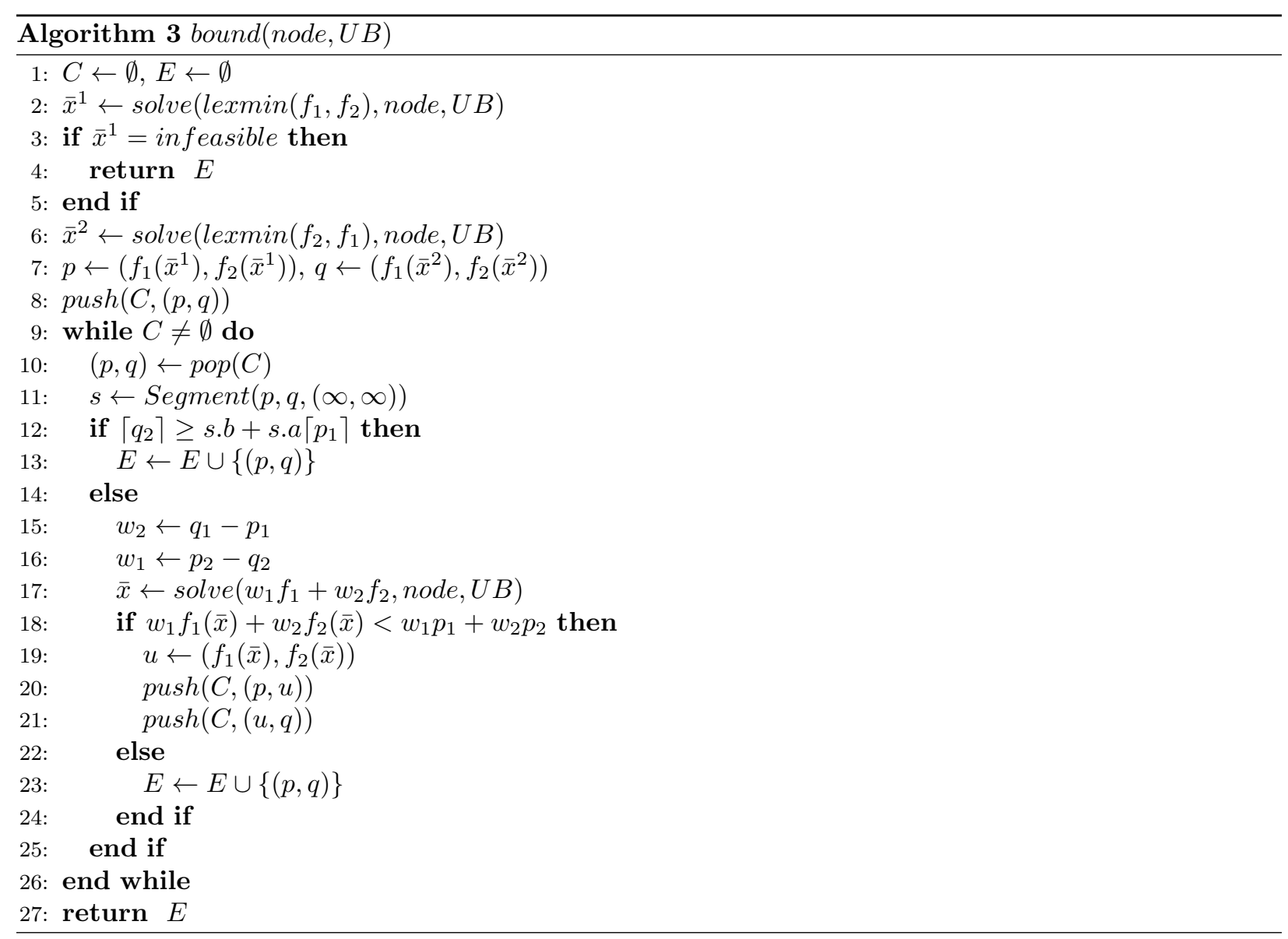


of Algorithm 3, this means that after retrieving $(p, q)$ from $C$ (line 10, Algorithm 3), we check if the triangle derived from $(p, q)$ does not contain any integer point.

Proposition 2 If the point $\left(\left\lceil p_{1}\right\rceil,\left\lceil q_{2}\right\rceil\right)$ is above the line connecting $p$ and q, i.e. $\left\lceil q_{2}\right\rceil \geq s . a\left\lceil p_{1}\right\rceil+s . b$, then the triangle derived from $(p, q)$ does not contain any integer point.

Proof 2 Since $p$ and $q$ are extreme points of the boundary of the convex hull, an additional LB point may only be found in the right triangle defined by the points $p,\left(p_{1}, q_{2}\right)$, q, with $p_{1} \leq q_{1}$ and $q_{2} \leq p_{2}$. Let us call $\left(p_{1}, q_{2}\right)$ the local ideal point. Since $\left\lceil p_{1}\right\rceil \geq p_{1}$ and $\left\lceil q_{2}\right\rceil \geq q_{2},\left(\left\lceil p_{1}\right\rceil,\left\lceil q_{2}\right\rceil\right)$ must either lie within the triangle or it is above the line connecting $p$ and $q$. Since any other integer point $z=\left(z_{1}, z_{2}\right)$ must have coordinates satisfying $z_{1} \geq\left\lceil p_{1}\right\rceil$ and $z_{2} \geq\left\lceil q_{2}\right\rceil$, if $\left(\left\lceil p_{1}\right\rceil,\left\lceil q_{2}\right\rceil\right)$ lies above the line connecting $p$ and $q$, no other integer point within this triangle exists. $\square$

In this case, the segment derived from $(p, q)$ is appended to $E$ in Algorithm 3 and lines 13-21 are skipped. Otherwise a weighted sum problem $\left(w_{1} f_{1}+w_{2} f_{2}\right)$ is solved, where $w_{1}$ and $w_{2}$ are determined by the algorithm. In the case where the resulting point $u$ lies below the line connecting $p$ and $q,(p, u)$ and $(u, q)$ are stored in $C$ for further processing, as in the algorithm by Aneja and Nair (1979). This enhancement is called lower bound lifting.

\subsection{Integer dominance}

Third, it is also possible to exploit the integrality of objective values for feasible integer solutions when branching on objective space. If we are in the situation of branching on objective space, it is typically because part of the LB set has been dominated by the UB set. In that case, we already know that the given local nadir point of any LB segment is in fact also dominated by the UB set (this is actually how this local nadir point is calculated, see Algorithm 1). So this local nadir point can be tightened. Figure 6 illustrates this principle: after filtering the LB segment $s$ with points from the upper bound set, $u$ and $v$ (Figures 6(a) and $6(\mathrm{~b})$, we obtain a reduced segment $s^{\prime}$. The new local nadir point $s^{\prime} . c$ is actually dominated by both $u$ and $v$ (Figure 6(c)). Moreover, each point in the area which is in a darker shade (Figure 6(d) is either (i) dominated by $u$ and/or $v$ or (ii) not integer. Therefore we can disregard the whole area. This can be achieved by subtracting any value $\rho$ such that $0 \leq \rho<1$ from both coordinates of the local nadir point $s^{\prime} . c$ after filtering, thus producing an improved segment $s^{\prime \prime}$. In order to implement this idea in our algorithm, we simply subtract $\rho$ from both coordinates of each upper bound point $u$ used in Algorithm 1 . This enhancement is called integer dominance. It is in fact similar to using an $\epsilon$ value of 1 in the $\epsilon$-constraint framework, or in the balanced box method.

\section{Computational study}

We investigate the efficiency of the proposed algorithm and enhancements through experimentation.

A generic version of BIOBAB is developed in Python 2.7 and Gurobi 6.5.0 is used to solve LPs and MIPs. Algorithms are run on an Intel Xeon E5-2650v2 CPU at $2.6 \mathrm{GHz}$, with a $4 \mathrm{~GB}$ memory limit and a two-hour CPU time limit. Except when explicitly stated otherwise, BIOBAB uses the linear relaxation of the original IP to compute lower bound sets. In terms of tree exploration, BIOBAB always performs a breadth-first search. Like in Boland et al. (2015a), we use performance profiles to compare the performances of different algorithms (Dolan and Moré (2002)). The performance of an algorithm for a certain instance is the ratio of the CPU time required by that algorithm for that instance to the best CPU time for that instance. The $x$-axis represents performance, while the $y$-axis represents the fraction of instances solved at a certain performance or better. If an algorithm does not terminate (i.e. find the Pareto set) for a certain instance, then it does not offer a performance for that instance. If no algorithm terminates for a given instance, then this instance is discarded.

In the following, we first describe the considered problems and benchmark instances for the first set of experiments, where we evaluate the different components of the algorithm and the impact of the integralitybased improvements. We then compare the results of our method with those of criterion space search 
Obj. 2

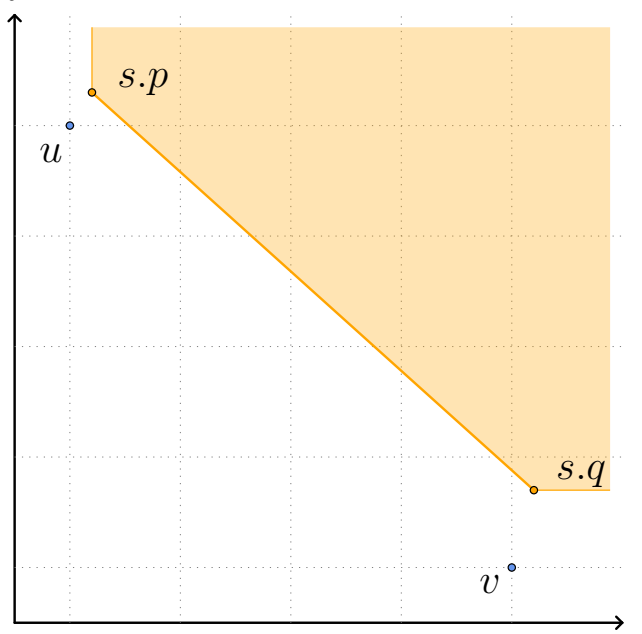

Obj. 1

(a)

Obj. 2

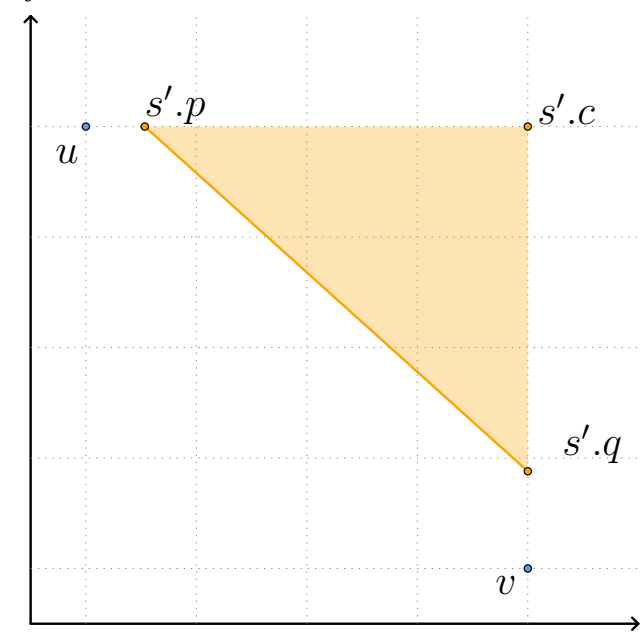

Obj. 1

(c)

\section{Obj. 2}

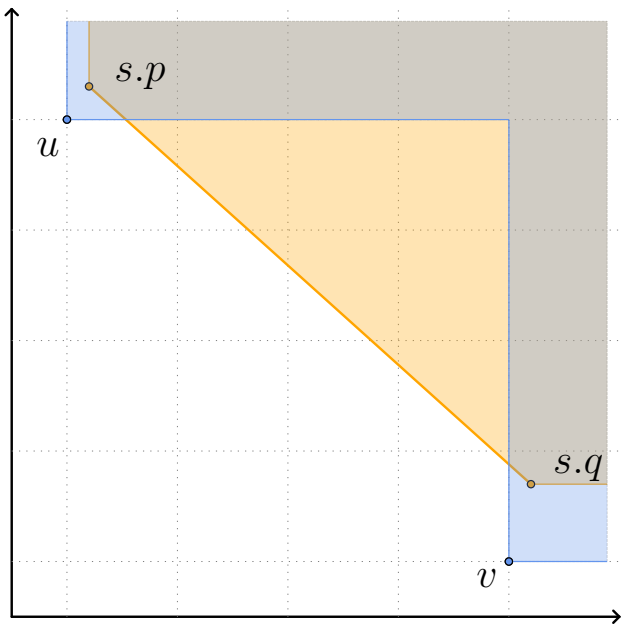

Obj. 1

(b)

\section{Obj. 2}

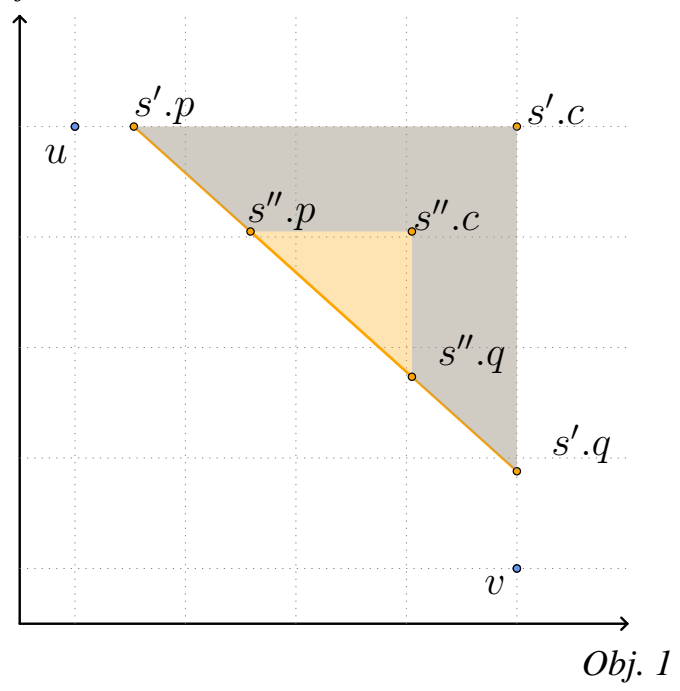

(d)

Figure 6: Part of a LB segment $s$ is either still dominated by the UB (represented by $u$ and $v$ ) or not containing any integer vector (darker shaded area in the fourth picture). 
algorithms and to an existing branch-and-bound method. Thereafter, we formally introduce the bi-objective team orienteering problem with time windows (BITOPTW), whose linear relaxation is solved by means of column generation, we explain the different components tailored to this problem, and we report the obtained results.

\subsection{Considered problems and benchmark instances}

For our first set of experiments, we apply BIOBAB to two different uncapacitated bi-objective facility location problems. The first uncapacitated facility location problem, which we call uncapacitated bi-objective facility location problem (UBOFLP), considers the cost of opening facilities as first objective and the coverage of the population as second objective. Population from a certain node can only be covered by an open facility if the distance between the two is below a threshold. For the UBOFLP, we use the data that was also used to generate instances from the bi-objective stochastic covering tour problem presented in Tricoire et al. (2012). The following simplifications are performed: (i) the transportation costs are ignored, (ii) the stochastic aspect is ignored and (iii) the distance aspect is simplified so that a node is either entirely covered or not covered at all by an open facility. In order to produce instances with various levels of difficulty, we concatenate the original instances: the $k^{\text {th }}$ UBOFLP instance consists of the data of the first $k$ covering tour problem instances. In total there are 36 UBOFLP instances, including 21 to 500 locations. The second uncapacitated facility location problem we consider is a simplification of the single-source capacitated facility location problem, where the capacity constraints are relaxed. The first objective is to minimize the cost of opening facilities, while the second objective is to minimize assignment costs. We name this problem SSUFLP from now on. We use the 120 instances publicly available from Gadegaard et al. (2016b). For both UBOFLP and SSUFLP, decision space branching is performed in a generic way. First, the average value of each binary variable over the whole LB set (or rather subset, see Section 4.4) is computed. Then, the variable with average value closest to 1 is selected for binary branching on that subset. We provide mathematical formulations for UBOFLP and SSUFLP in Appendices B and C For each of these problems, we compute the greatest common divisor for each objective and use it as valid $\epsilon$ value in the various methods. Since all costs are integer, $\epsilon$ is always at least 1 .

We provide information on the cardinality of efficient sets for UBOFLP and SSUFLP instances in Figure 7.

\subsection{Impact of integrality-based improvements}

In a first series of experiment, we assess the efficiency of the improvements described in Section 5 , using the UBOFLP and SSUFLP data set. We run a version of BIOBAB with no improvement at all, a version with objective space branching only, a version with segment tightening only, a version with lower bound lifting only, and a version with all three improvements. Performance profiles are displayed in Figure8 8 On UBOFLP instances, full BIOBAB as well as segment tightening clearly outperform the other variants. Whether full BIOBAB outperforms the version with segment tightening only is unclear, because the whole chart is scaled to also include the performance profiles of poorly performing variants. For the same reason, it is also unclear whether lower bound lifting brings a significant improvement over bare BIOBAB. On SSUFLP instances, the differences are smaller but still clearly marked; full BIOBAB is still the best, but this time it appears that objective-space branching is working better on its own than segment tightening. Again, it is unclear whether lower bound lifting brings any improvement.

We further investigate the individual contributions of the various BIOBAB components, by comparing full BIOBAB to full BIOBAB minus individual improvements. We first look at segment tightening, which appears to be crucial in certain cases, as shown in Figure 8 Figure 9 presents the performance of full BIOBAB with and without segment tightening. Approximately $70 \%$ of UBOFLP instances cannot be solved within two hours when segment tightening is disabled, which makes it a crucial component. All SSUFLP Instances can be solved, but the profile of full BIOBAB is still consistently better than the profile without segment tightening. Full BIOBAB is up to $56 \%$ faster.

We now also look at the performance profile of full BIOBAB, versus full BIOBAB minus lower bound 


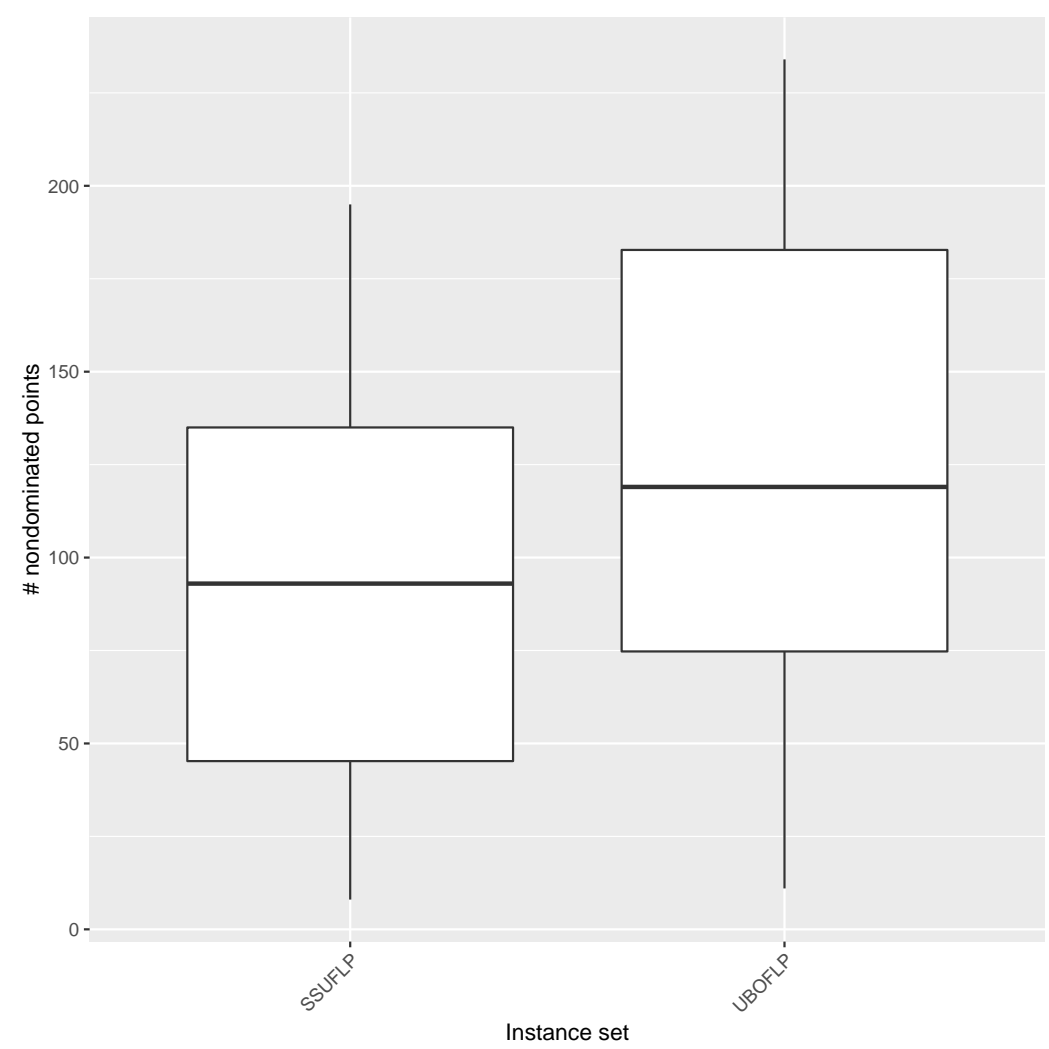

Figure 7: Number of non-dominated points for SSUFLP and UBOFLP instance sets.

SSUFLP: 120 instances.

UBOFLP: 36 instances.

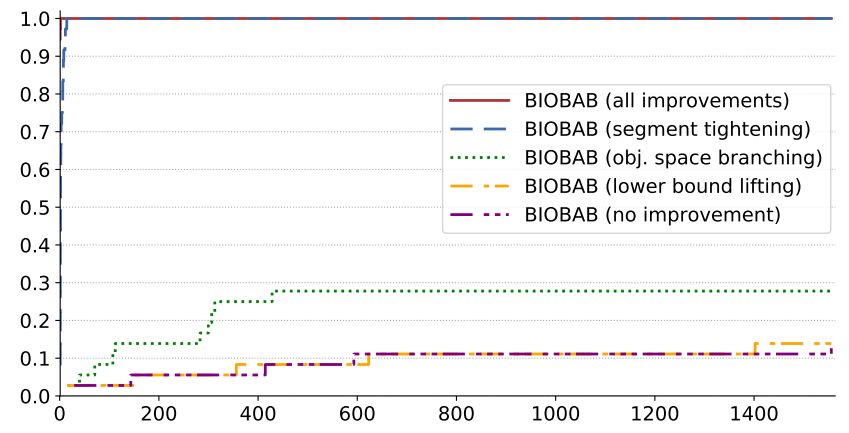

(a) UBOFLP

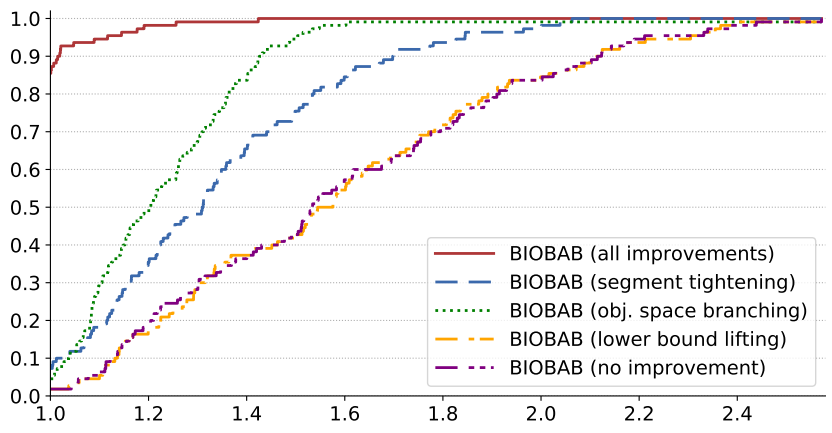

(b) SSUFLP

Figure 8: BIOBAB: individual contribution of various improvements 


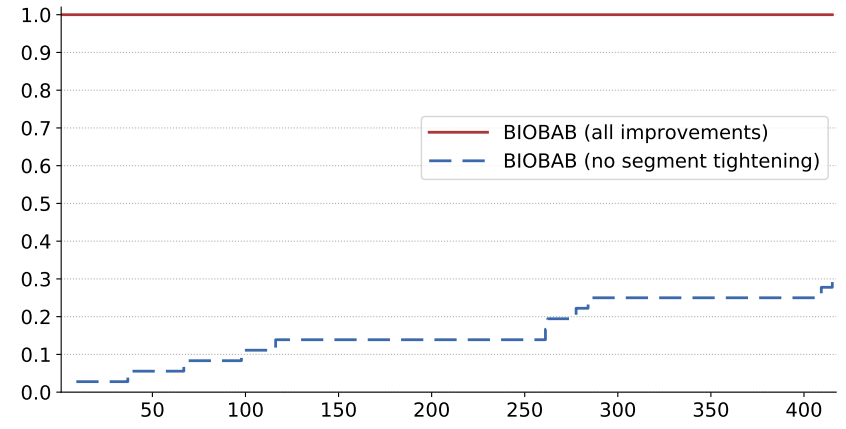

(a) UBOFLP

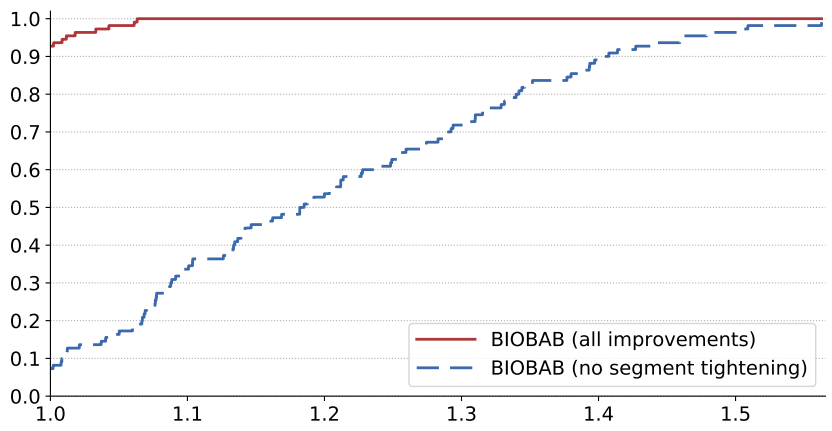

(b) SSUFLP

Figure 9: Full BIOBAB vs no segment tightening

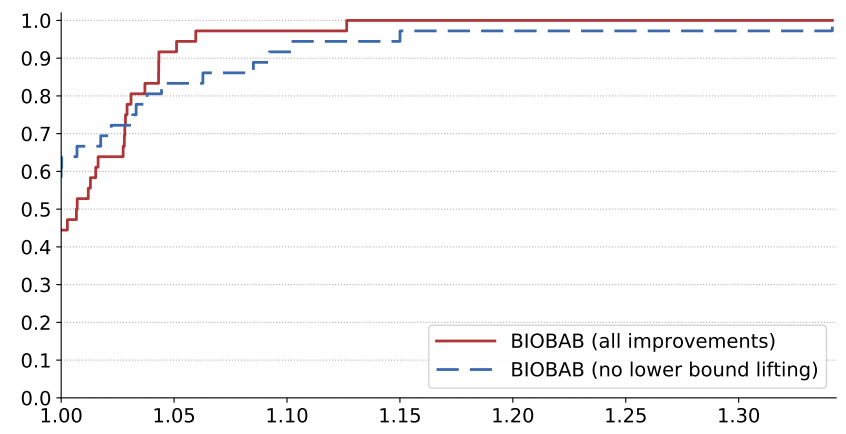

(a) UBOFLP

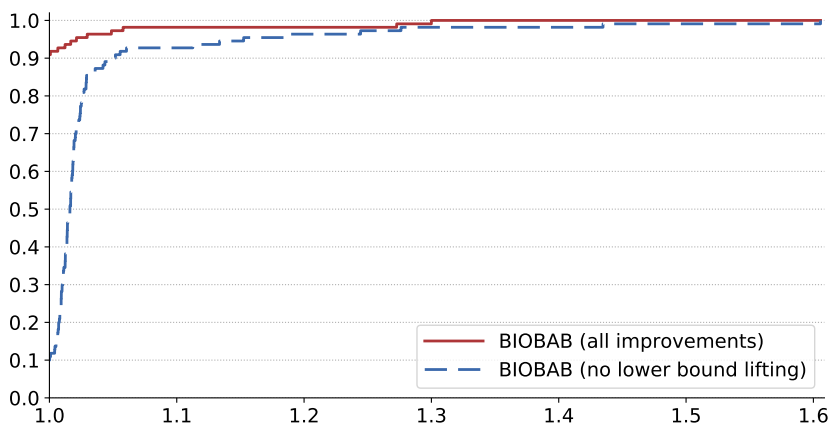

(b) SSUFLP

Figure 10: Full BIOBAB vs no lower bound lifting

lifting, depicted in Figure 10. The impact of lower bound lifting is less spectacular, but still significant. In rare cases not using lower bound lifting actually improves performance, but only very slightly (less than $5 \%$, on some UBOFLP instances). Lower bound lifting speeds up the algorithm by up to $34 \%$ on UBOFLP instances and by up to $60 \%$ on SSUFLP instances. As mentioned earlier, lower bound lifting only involves constant time operations, so the overhead is minimal and will never cause an important decrease in performance, even when it is entirely useless. Therefore it is safe to use it in general.

Full BIOBAB is compared to the variant without OSB in Figure 11. The impact of objective space branching is important: using it speeds up BIOBAB by a factor up to 14.7 for UBOFLP instances and up to 2.06 for SSUFLP instances. We note here that it is not surprising that objective space branching works well when combined with segment tightening, since segment tightening creates discontinuities in LB sets, thus allowing objective space branching to be applied.

These performance profiles clearly show that every algorithmic improvement brings added value, with rare exceptions for lower bound lifting. In order to provide additional insight on the use of objective space branching, we consider additional information related to full BIOBAB and full BIOBAB minus OSB: we analyze CPU time, number of nodes explored during tree search and number of linear programs solved during the whole search. Table 1 shows the per-instance difference between the two versions of BIOBAB, on UBOFLP instances. We note that the total number of nodes explored does not vary that much between both methods, but the number of LPs solved does: the version without objective space branching solves more than ten times more LPs in some cases. We believe this validates the idea that branching on objective space avoids visiting several times regions that have already been established to be dominated by the UB set; apparently visiting these regions requires solving many LPs. 


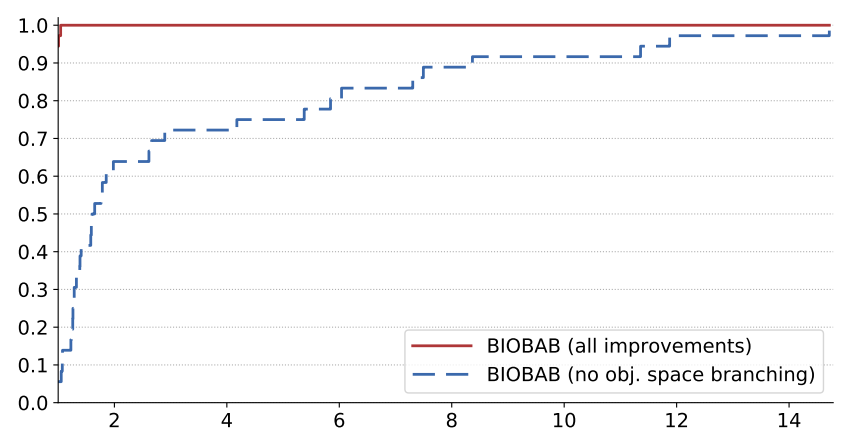

(a) UBOFLP

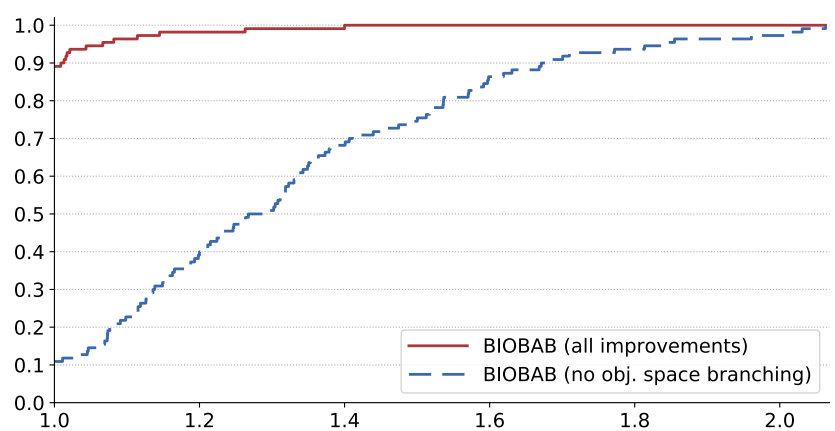

(b) SSUFLP

Figure 11: Full BIOBAB vs no objective space branching

\begin{tabular}{|c|c|c|c|c|c|c|}
\hline \multirow[b]{2}{*}{ Instance } & \multicolumn{3}{|c|}{ Full BIOBAB } & \multicolumn{3}{|c|}{ No obj. space branching } \\
\hline & \# LPs & \# nodes & CPU (s) & \# LPs & \# nodes & CPU (s) \\
\hline 021 & 23 & 1 & 0.30 & 23 & 1 & 0.28 \\
\hline 044 & 59 & 3 & 0.57 & 59 & 3 & 0.61 \\
\hline 055 & 73 & 3 & 0.89 & 73 & 3 & 0.85 \\
\hline 072 & 95 & 3 & 1.59 & 95 & 3 & 1.56 \\
\hline 090 & 135 & 7 & 3.04 & 197 & 7 & 4.18 \\
\hline 106 & 157 & 7 & 5.07 & 235 & 7 & 6.33 \\
\hline 120 & 173 & 7 & 6.44 & 267 & 7 & 8.88 \\
\hline 132 & 193 & 9 & 8.30 & 335 & 9 & 13.52 \\
\hline 134 & 197 & 9 & 8.58 & 339 & 9 & 15.16 \\
\hline 135 & 199 & 9 & 8.41 & 333 & 7 & 14.72 \\
\hline 162 & 263 & 11 & 16.78 & 401 & 9 & 26.39 \\
\hline 182 & 466 & 17 & 32.53 & 699 & 13 & 51.58 \\
\hline 203 & 478 & 17 & 41.57 & 697 & 11 & 66.53 \\
\hline 226 & 255 & 9 & 31.41 & 319 & 9 & 44.19 \\
\hline 254 & 259 & 9 & 41.72 & 335 & 9 & 53.46 \\
\hline 264 & 269 & 9 & 47.21 & 345 & 9 & 58.12 \\
\hline 266 & 271 & 9 & 49.48 & 347 & 9 & 63.44 \\
\hline 275 & 277 & 9 & 53.35 & 303 & 7 & 56.18 \\
\hline 294 & 285 & 9 & 64.19 & 361 & 9 & 84.88 \\
\hline 295 & 277 & 7 & 71.97 & 363 & 9 & 88.12 \\
\hline 296 & 289 & 9 & 74.64 & 365 & 9 & 94.16 \\
\hline 326 & 361 & 15 & 112.16 & 1459 & 29 & 467.91 \\
\hline 342 & 399 & 19 & 137.47 & 1125 & 15 & 397.68 \\
\hline 355 & 473 & 31 & 165.05 & 3509 & 61 & 1379.35 \\
\hline 370 & 455 & 25 & 195.29 & 983 & 19 & 386.76 \\
\hline 388 & 461 & 21 & 204.75 & 3159 & 39 & 1494.41 \\
\hline 399 & 531 & 35 & 236.88 & 1449 & 33 & 630.14 \\
\hline 410 & 595 & 45 & 297.69 & 1061 & 17 & 551.82 \\
\hline 428 & 645 & 51 & 342.32 & 7453 & 89 & 3885.49 \\
\hline 436 & 609 & 43 & 351.86 & 1585 & 31 & 919.27 \\
\hline 449 & 679 & 53 & 400.70 & 10586 & 183 & 5891.94 \\
\hline 458 & 713 & 57 & 432.92 & 5387 & 85 & 3241.36 \\
\hline 472 & 719 & 59 & 462.29 & 4681 & 113 & 2790.82 \\
\hline 482 & 821 & 75 & 591.75 & 5511 & 81 & 3456.99 \\
\hline 499 & 773 & 67 & 542.06 & 4209 & 97 & 2912.71 \\
\hline 500 & 759 & 65 & 583.60 & 9003 & 135 & 6927.28 \\
\hline
\end{tabular}

Table 1: Effect of objective space branching on tree search (UBOFLP instances). 


\begin{tabular}{cccc}
\hline Problem & \# root & \# other & max. depth \\
\hline SSUFLP & 23.84 & 2.07 & 12.24 \\
UBOFLP & 5.37 & 0 & 0 \\
\hline
\end{tabular}

Table 2: Additional information on objective-space branching: average values. \# root: number of OSB branches at the root node \# other: number of OSB branches at other nodes max. depth: maximum depth at which OSB occurred

We now look closer at the number of branches generated by OSB. For that purpose we count, for each instance solved, how many branches are generated by OSB. We differentiate the number of branches generated at the root node and in the remainder of the branch-and-bound tree. We report average values for these two indicators, as well as the maximum tree depth at which OSB happened, in Table 2. Instances that are entirely solved without branching are disregarded. For both problem classes, OSB generates more branches at the root node, thus indicating that integer solutions are already found while solving the root node. For UBOFLP, OSB actually only happens at the root node; for SSUFLP, the average number of branches at nodes other than the root node is very close to 2 , which suggests that it typically happens when one new efficient point is found. We also observe that a lower number of branches at a given node does not necessarily indicate that fewer integer solutions are found at this node, since segment tightening can also eliminate the objective space between two integer points, thus reducing the number of branches. But if there are $k$ OSB branches following the bounding procedure at a given node, then we know that at least $k-1$ integer solutions were found during this bounding procedure.

\subsection{Comparison with criterion space search algorithms}

We implemented a generic balanced box method as described in Boland et al. (2015a) (it is sketched in Algorithm 5 in the Appendix), including solution harvesting and other enhancements, within the same Python code base as BIOBAB. The frequency with which solution harvesting is called is controlled using parameter $\beta$. Like in the original paper, we set $\beta=0.15$ following preliminary experiments. Also like in the original paper, we find that in order to produce lexicographic minima, solving explicitly two successive IPs is more efficient than solving one weighted-sum IP. Previously found integer solutions that are valid for the problem at hand (i.e. within the same rectangle) are used to provide a cutoff value to the MIP solver.

Within the same code base we also develop generic versions of the $\epsilon$-constraint framework (see Algorithm 4 in the Appendix) as well as the bi-directional $\epsilon$-constraint framework described in Boland et al. (2015a). We note here that there are always two ways to run the single-directional $\epsilon$-constraint algorithm, either starting with objective 1 or with objective 2 . For a given problem class, one of these two ways is typically faster than the other, sometimes much faster. In these cases, it is likely that the bi-directional version will not be as fast since it has to solve IPs in the slower direction for $50 \%$ of these IPs. All $\epsilon$-constraint methods benefit from the applicable enhancements developed for the balanced box method.

We first compare BIOBAB and other criterion space search methods on the UBOFLP. We note here that BIOBAB solves the linear relaxation of the UBOFLP to compute LB sets, whereas criterion space search methods call the MIP solver directly. Performance profiles are given in Figure 12. BIOBAB appears to perform better than criterion space search methods on the UBOFLP. It is however not overwhelming, as all methods always converge within a factor 3 of the best performance overall, i.e. within the same order of magnitude. Nonetheless, BIOBAB is definitely competitive on these instances. We also note that single-directional $\epsilon$-constraint performs better than its bi-directional counterpart.

We now look at the performance profiles of the same methods, this time on SSUFLP instances. These profiles are represented in Figure 13. The picture is now different: BIOBAB is the worst of all compared methods. It is however, still in the same order of magnitude ( 7 times slower than the best method in the worst case). In this comparison, all criterion space search methods benefit from the power of commercial MIP solvers, which are especially efficient at tree exploration and branching on decision variables. Even 


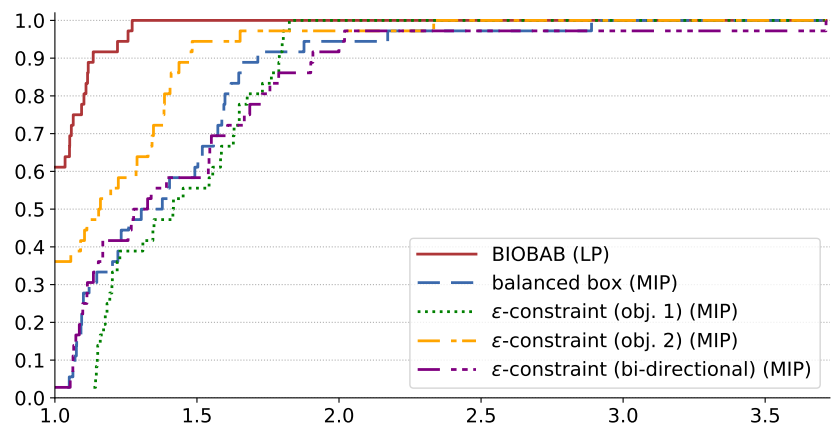

Figure 12: UBOFLP: BIOBAB vs criterion space search methods

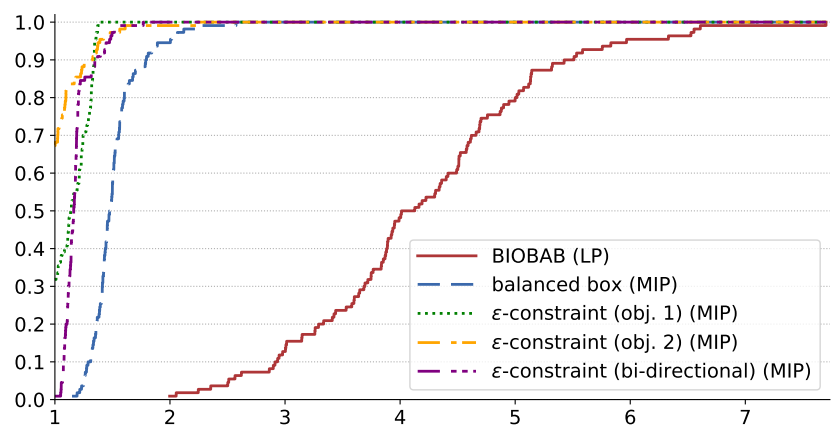

Figure 13: SSUFLP: BIOBAB vs criterion space search methods

though we show above that objective space branching significantly improves the performance of BIOBAB, branching on decision variables is still performed in a somewhat naive way (fractional variable with value closest to 1 , breadth-first tree exploration). In the next experiment we do not relax integrality constraints when computing LB sets for the SSUFLP: IPs are solved within the bounding procedure of BIOBAB. An expectation is that LB sets will be of better quality, at the cost of extra CPU time. Better LB sets should reduce the size of the branch-and-bound tree of BIOBAB, thus delegating part of the task of tree search to the commercial MIP solver. Another expectation is that more integer solutions will be obtained earlier in the search, which in turn will allow OSB and integer dominance to speed up the search. We now compare this version of BIOBAB to the same criterion space search methods, in Figure 14 . The performance profiles clearly show that using an IP instead of its linear relaxation can be beneficial: BIOBAB is now performing much better, actually slightly better than all criterion space search methods.

Additionally, we display in Figure 15 the total number of linear programs (LPs) solved by each algorithm to generate the whole set of non-dominated points. It is clear that the poor performance of BIOBAB using LPs is correlated with the unusually high amount of LPs it requires to solve, while BIOBAB using MIPs requires to solve a number of LPs comparable with that of other algorithms. This strengthens the idea that efficient branching on decision variables is the issue here.

\subsection{Comparison with bi-objective branch-and-bound}

We now compare BIOBAB with the branch-and-bound algorithm for bi-objective mixed-integer programs from Belotti et al. (2013). Their algorithm is for mixed-integer programs, but they also provide results on the bi-objective set covering problem, which only uses binary variables. We run BIOBAB on the same instances and can therefore compare the CPU time required for solving these instances. We present results using the same format as in their article. Their computer is a $3.2 \mathrm{GHz}$ workstation with $4 \mathrm{~GB}$ of RAM. We observe 


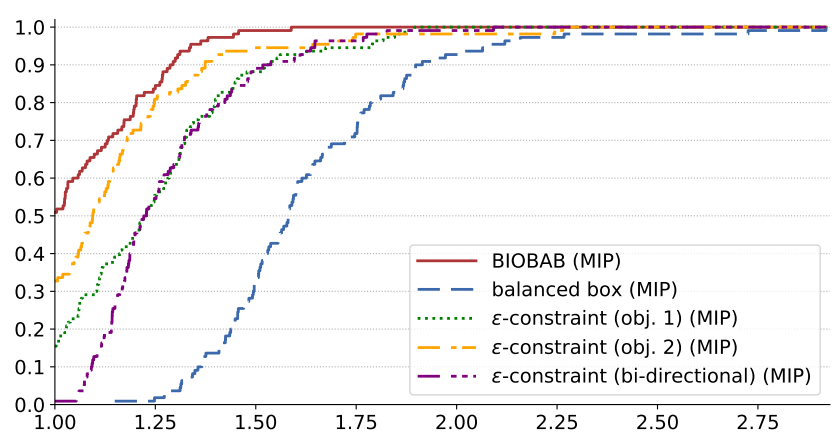

Figure 14: SSUFLP: BIOBAB (MIP) vs criterion space search methods

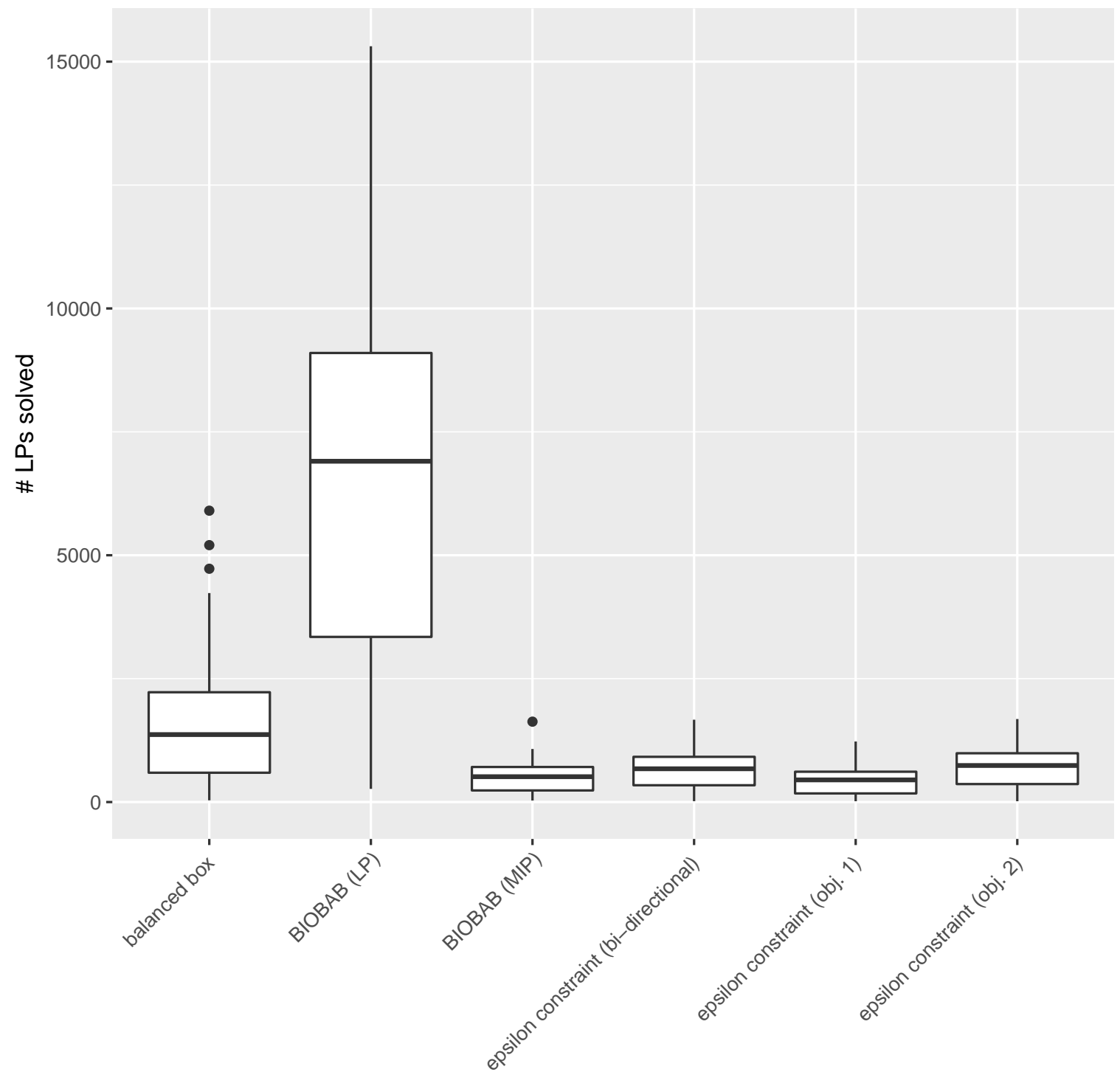

Algorithm

Figure 15: SSUFLP: number of LPs solved by each algorithm. 


\begin{tabular}{llll}
\hline Instance size $(m \times n)$ & $\#$ instances & CPU $(\mathrm{s})$ Belotti et al. & CPU $(\mathrm{s})$ BIOBAB \\
\hline $5 \times 25$ & 1 & 0.1 & 0.01 \\
$10 \times 50$ & 1 & 2.1 & 0.73 \\
$10 \times 100$ & 4 & 49.3 & 3.76 \\
\hline
\end{tabular}

Table 3: Comparison of BIOBAB and bi-objective branch and bound from Belotti et al. (2013) on biobjective set covering problem instances: average CPU time. Instances have $m$ variables and $n$ constraints. Belotti et al.: $3.2 \mathrm{GHz}$ workstation with $4 \mathrm{~GB}$ of RAM. BIOBAB: $2.6 \mathrm{GHz}$ Xeon CPU with a 4 GB RAM limit.

that BIOBAB is always faster, more than 10 times faster for the largest instances. Even if it is not clear how much has to attributed to differences in CPU, we can safely conclude that BIOBAB can compete with a state-of-the-art branch-and-bound method on these instances.

\subsection{Application to cases which cannot benefit from the power of MIP solvers}

A major strength of state-of-the-art MIP solvers lies in their ability to conduct efficient branch-and-bound or branch-and-cut tree search, through involved tree exploration heuristics that are industrial secrets. However, it is not always possible to benefit from these advanced methods. Sometimes even the single objective problem version is too hard to solve with a MIP solver. We want to assess how BIOBAB fares in such cases. For that purpose, we use the BITOPTW as test case. The BITOPTW is formally described in Appendix D. Such a model is challenging for current state-of-the-art MIP solvers.

\subsubsection{Lower bound set: column generation}

Since state-of-the-art exact methods for single-objective routing problems mostly rely on column generation based techniques (see, e.g. Baldacci et al. 2012), we also generate lower bound sets for the BITOPTW by means of column generation.

Let $P_{r}$ denote the total score or profit achieved by route $r, C_{r}$ the total travel cost of route $r$, and let $a_{i r}$ indicate whether location $i$ is visited by route $r\left(a_{i r}=1\right)$ or not $\left(a_{i r}=0\right)$. Using binary variables $x_{r}$ equal to 1 if route $r$ is selected from the set $\Omega$ of all feasible routes, the BITOPTW can also be formulated as a path-based model:

$$
\begin{array}{cc}
f_{1}(x)=\min \sum_{r \in \Omega} C_{r} x_{r} & \\
f_{2}(x)=\max \sum_{r \in \Omega} P_{r} x_{r} & \\
\sum_{r \in \Omega} a_{i r} x_{r} \leq 1 & \forall i \in N \\
\sum_{r \in \Omega} x_{r}=m & \\
x_{r} \in\{0,1\} & \forall r \in \Omega .
\end{array}
$$

Relaxing integrality requirements on the $x_{r}$ variables, we replace constraints $(6]$ with:

$$
x_{r} \geq 0 \quad \forall r \in \Omega
$$

We also combine the two objective functions into a weighted sum ( $w_{1}$ and $w_{2}$ giving the respective nonnegative weights determined by our LB set computation procedure bound):

$$
\min \sum_{r \in \Omega}\left(w_{1} C_{r}-w_{2} P_{r}\right) x_{r}
$$


We thus obtain a single objective linear problem that can be solved by means of column generation, which allows us to compute LB sets using Algorithm 3 . In column generation (see, e.g. Desrosiers and Lübbecke 2005), in each iteration a subset of promising columns is generated and appended to the restricted set of columns $\Omega^{\prime}$ and the single objective linear program is re-solved on $\Omega^{\prime}$. Column generation continues as long as new promising columns exist. Otherwise, an optimal solution has been found (i.e. in that case, an optimal solution of the single objective linear program on $\Omega^{\prime}$ is also an optimal solution of the single objective linear program on $\Omega$ ). Promising columns are identified using dual information. Let $\pi_{i}$ denote the dual variable associated with constraint (4) for a given $i$ and $\alpha$ the dual variable associated with constraint (5), the pricing subproblem we have to solve corresponds to:

$$
\min w_{1} C_{r}-w_{2} P_{r}-\sum_{i \in N} a_{i r} \pi_{i}-\alpha
$$

subject to constraints (27)-(34) given in Appendix D, omitting subscript $k$. It is an elementary shortest path problem with resource constraints that can be solved by means of a labeling algorithm (cf. Feillet et al. 2004). In our labeling algorithm, a label carries the following information: the node the label is associated with, the time consumption until that node, the reduced cost so far, which nodes have been visited along the path leading to the node, and a pointer to the parent label. In order to compute the reduced cost of the path associated with a given label, we use a reduced cost matrix that is generated before the labeling algorithm is called. The reduced cost of $\operatorname{arc}(i, j)$ is $\bar{c}_{i j}=w_{1} c_{i j}-w_{2} S_{i}-\pi_{i}$, where if $i=0, \pi_{i}$ is replaced by $\alpha$ and $c_{i j}$ gives the costs for traversing arc $(i, j)$ and $S_{i}$ the score for visiting location $i$. Since the aim of this paper is not to investigate the most efficient pricing algorithm, we refrain from adopting all enhancements proposed in the literature (e.g. Righini and Salani 2009).

Note that during the execution of BIOBAB we keep all previously generated columns in the column pool. We only temporarily deactivate those columns that are incompatible with current branching decisions. When branching on objective space, it can happen that the current pool of columns does not allow to produce a feasible solution. This can be due to two reasons: either (i) because there exists no feasible solution to the current problem or (ii) because there exists a feasible solution but it cannot be reached with the currently available columns. In order to fix this issue and guarantee feasibility, we use a dummy column which allows to satisfy every constraint from the current problem, including the branching decisions described in Section 6.5.2. These include branching on control points. Therefore, some control points may be mandatory and others may be forbidden. Thus, the dummy column covers all mandatory control points, does not cover any forbidden control point, uses all the available vehicles, has a cost inferior to the maximum allowed cost and a profit superior to the minimum allowed profit. Using this column is penalized in the objective function, so that the column generation procedure converges to feasible solutions that do not use the dummy column. Assuming the variable associated to this dummy column is $x_{D}$, the objective function described in Equation 8 is modified as follows:

$$
\min \sum_{r \in \Omega}\left(w_{1} C_{r}-w_{2} P_{r}\right) x_{r}+M x_{D},
$$

where $M$ is an arbitrarily large number. The dummy column is only activated when no feasible solution can be found, and it is systematically deactivated after column generation converges. At this point, if $x_{D}$ has a strictly positive value then there does not exist a feasible solution that satisfies all the branching decisions.

\subsubsection{Branching scheme}

Objective space branching is performed as usual (see Section 4.4). Since objective space branching involves setting bounds on both objectives, we include these constraints right from the start (initially setting the 
respective bounds to infinity) and later update them according to the branching decisions:

$$
\begin{gathered}
\sum_{r \in \Omega} C_{r} x_{r} \leq c_{1}, \\
\sum_{r \in \Omega}-P_{r} x_{r} \leq c_{2},
\end{gathered}
$$

where $c=\left(c_{1}, c_{2}\right)$ is the local nadir point. In order to properly incorporate dual information from these two constraints into the subproblem, we modify the reduced cost matrix. Let $\lambda_{1}$ be the dual variable value associated with constraint (11) and $\lambda_{2}$ the value associated with constraint (12), then the reduced cost of a given arc $(i, j)$ is given by $\bar{c}_{i j}^{\prime}=\left(w_{1}-\lambda_{1}\right) c_{i j}-\left(w_{2}+\lambda_{2}\right) S_{i}-\pi_{i}$. Regarding decision-space branching, we either branch on control points or on arcs (Line 4 in Algorithm 2). First, we check if a control point is visited a fractional number of times. Since each lower bound represents a set of solutions, the number of times a control point is visited may take different values within the same LB set. In order to select a control point to branch on, we consider the supported (fractional) solutions defining the current LB set. We then average the number of visits for each control point over this set of solutions. The control point with the average number of visits closest to 0.5 is then selected for branching. In the case where each control point is visited 0 or 1 time on average, we check for arcs that are traversed a fractional number of times on average, following the same procedure. Branching on control points can be achieved by modifying constraint (4) in the master problem. In order to force the visit of control point $i$, this constraint becomes $\sum_{r \in \Omega} a_{i r} x_{r} \geq 1$.

In order to forbid the visit of $i$, this constraint becomes $\sum_{r \in \Omega} a_{i r} x_{r} \leq 0$. As is usual in branch-and-price for routing, branching on arcs involves adding constraints to the subproblem.

\subsubsection{Applying the $\epsilon$-constraint method}

In order to asses the quality of our BIOBAB on the BITOPTW, we compare it to the $\epsilon$-constraint method, using the faster direction. In this case, the function solveMIP (Line 4 of Algorithm 4 in the Appendix) uses the same tree search algorithm as BIOBAB, i.e. the same rules for branching are applied, with the exception of objective space branching, since a single-objective problem is solved. The bounding procedure is similar to the one used in BIOBAB, i.e. the same column generation code is used. However, instead of solving a succession of LPs to compute a LB set, a single solution is produced in each call to the procedure. Other than that, the code is exactly the same for both methods. This means that in the $\epsilon$-constraint framework, previously generated columns are reused. Additionally, a single-objective solution to the linear relaxation yields a LB segment ; therefore, the existing UB set can be used to fathom subtrees using the filtering procedure described in Section 4.3. This works in combination with the fact that all produced integer solutions are stored, thus enhancing the $\epsilon$-constraint implementation with improvements developed for BIOBAB.

\subsubsection{BITOPTW Benchmark instances}

We use the TOPTW instances by Righini and Salani (2009) and we reduce their size by considering the first 15, 20, 25, 30 and 35 customers only. Using instances c101_100, r101_100, rc101_100 and pr01 and the number of customers mentioned above, we generate test instances for 1, 2, 3 and 4 vehicles. In total there are 80 BITOPTW instances.

\subsubsection{Computational results}

We now compare BIOBAB with the $\epsilon$-constraint method. Column generation can be time-intensive, so in this case all procedures are implemented in $\mathrm{C}++$. The same column generation code is used by both BIOBAB and $\epsilon$-constraint. We run both algorithms on the same computer as mentioned above, and produce the performance profile depicted in Figure 16. As we can see, BIOBAB offers a better performance overall. Although the $\epsilon$-constraint method is within the same order of magnitude for almost $90 \%$ of the instances, 


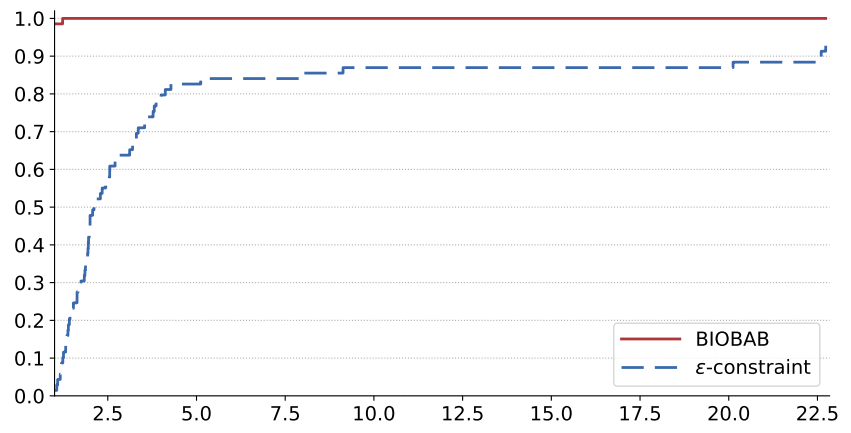

Figure 16: BIOBAB vs $\epsilon$-constraint when using column generation to solve the underlying linear program.

which is good, it can get up to 20 times slower in some rare cases. More strikingly, there are instances solved by BIOBAB that cannot be solved within the allotted CPU budget by the $\epsilon$-constraint method.

\section{Conclusion}

We have introduced a new algorithm for bi-objective integer optimization. The algorithm is a generalization of single-objective branch-and-bound to the bi-objective context. Our algorithm is generic and uses a branching rule exploiting the bi-objective nature of the underlying optimization problem. We have also developed several enhancements based on the integrality of the objective values of efficient solutions. Experiments show that these enhancements significantly improve the performance of BIOBAB, and make it competitive with state-of-the-art generic methods for bi-objective optimization for integer programs. In order to demonstrate the versatility of our framework, we have also applied it to a routing problem where the lower bound linear program is solved by means of column generation, thus providing, to the best of our knowledge, the first bi-objective branch-and-price algorithm. Standard criterion space search methods rely on the efficiency of MIP solvers and show their limitations in such cases: the proposed branch-and-bound algorithm outperforms the $\epsilon$-constraint method, which is a standard benchmark for multi-objective optimization.

One research perspective lies in generalizing the concept of lower bound set to more than two objectives, thus allowing exact approaches relying on this notion for more than two objectives. However this will raise the issue of the cardinality of the efficient set, which typically increases exponentially with the number of objectives. Therefore another research perspective lies in deriving approximation methods for multi-objective optimization based on exact procedures, with the goal of sacrificing neither the quality of solutions nor the readability of the produced set of solutions.

\section{Acknowledgments}

We wish two thank four anonymous referees for their valuable comments. Furthermore, financial support from the Austrian Science Fund (FWF): P23589-N13 is gratefully acknowledged.

\section{A Algorithms for criterion space search methods}

Algorithms 4 and 5 outline the $\epsilon$-constraint method and the balanced box method, respectively. The set $P$ denotes the set of Pareto optimal solutions. Function solveMIP can either be a call to Gurobi or CPLEX or to a tailor-made branch-and-bound algorithm. The value of $\epsilon$ is supposed to be valid for the problem at hand; for instance with pure integer problems with integer coefficients for the objective function, $\epsilon=1$ is such a valid value. 

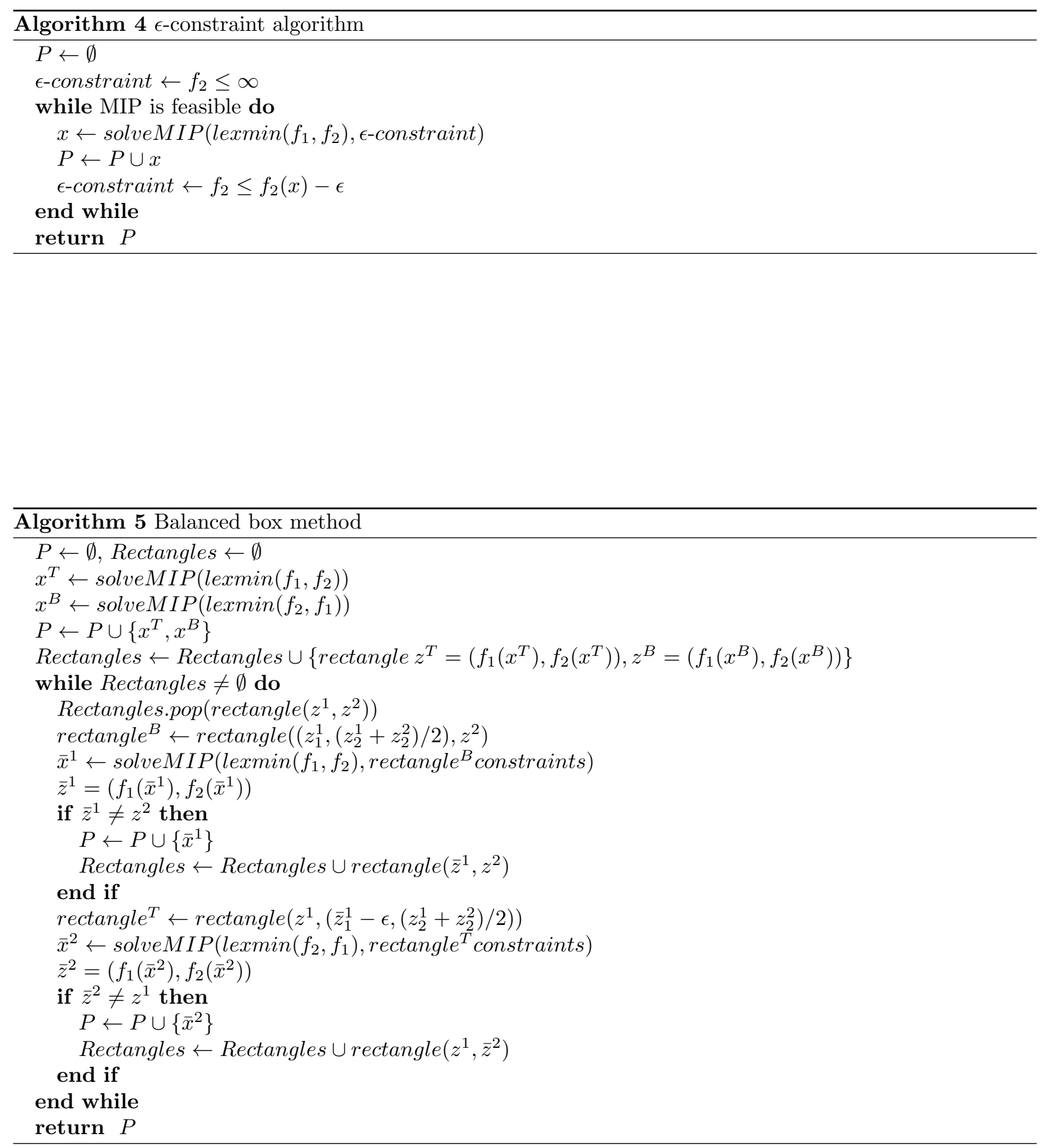


\section{B UBOFLP: Model formulation}

In the UBOFLP, we are given a set $V$ of potential facilities and a set $N$ of locations. Furthermore, we denote by $N_{i}$ the set of locations that can be covered by facility $i$ because they are within a certain radius. Each facility has opening costs $F_{i}$ and each location has a weight or demand $W_{j}$. The considered objectives simultaneously minimize the opening costs of facilities and maximize the total covered demand.

Using binary decision variables $y_{i} \in\{0,1\}$ equal to 1 if facility $i$ is opened and 0 otherwise, and $x_{i j} \in\{0,1\}$ equal to 1 if location $j$ is assigned to facility $i$, we formally define the UBOFLP as follows:

$$
\begin{aligned}
& \min \sum_{i \in V} F_{i} y_{i} \\
& \max \sum_{i \in V} \sum_{j \in N_{i}} W_{j} x_{i j}
\end{aligned}
$$

subject to:

$$
\begin{aligned}
x_{i j} & \leq y_{i} \\
\sum_{i \in V} x_{i j} & =1 \\
y_{i} & \in\{0,1\} \\
x_{i j} & \in\{0,1\}
\end{aligned}
$$

$$
\begin{aligned}
& \forall i \in V, j \in N, \\
& \forall j \in N, \\
& \forall i \in V, \\
& \forall i \in V, j \in N .
\end{aligned}
$$

\section{SSUFLP: Model formulation}

In the SSUFLP, we are given a set $V$ of potential facilities and a set $N$ of locations that have to be assigned to one of the facilities each. Each facility has opening costs $F_{i}$ and it costs $c_{i j}$ to assign location $j$ to facility $i$. The considered objectives simultaneously minimize the total opening costs of facilities and the total assignment costs.

Using binary decision variables $y_{i} \in\{0,1\}$ equal to 1 if facility $i$ is opened and 0 otherwise, and $x_{i j} \in\{0,1\}$ equal to 1 if location $j$ is assigned to facility $i$, we formally define the SSUFLP as follows:

$$
\begin{aligned}
& \min \sum_{i \in V} F_{i} y_{i} \\
& \min \sum_{i \in V} \sum_{j \in N} c_{i j} x_{i j}
\end{aligned}
$$

subject to:

$$
\begin{aligned}
x_{i j} & \leq y_{i} \\
\sum_{i \in V} x_{i j} & =1 \\
y_{i} & \in\{0,1\} \\
x_{i j} & \in\{0,1\}
\end{aligned}
$$

$$
\begin{aligned}
& \forall i \in V, j \in N, \\
& \forall j \in N, \\
& \forall i \in V, \\
& \forall i \in V, j \in N .
\end{aligned}
$$

\section{BITOPTW: Model formulation}

The BITOPTW is defined on a directed graph $G=(V, A)$, where $A$ is the set of arcs and $V$ the set of vertices, representing the starting location (vertex 0 ), the ending location (vertex $n+1$ ) and $n$ control points. Each control point $i$ is associated with a score $S_{i}$, a service time $d_{i}$ and a time window $\left[e_{i}, l_{i}\right]$. Each route $k \in K$, with $|K|=m$, has to start at location 0 and end at location $n+1$ and each arc $(i, j)$ is associated with travel 
cost $c_{i j}$ and travel time $t_{i j}$. The aim is to maximize the total collected score and to simultaneously minimize the total travel cost. Using binary decision variables $z_{i} \in\{0,1\}$ equal to 1 if location $i$ is visited and 0 otherwise, and $y_{i j k} \in\{0,1\}$ equal to 1 if $\operatorname{arc}(i, j)$ is traversed by route $k$ and 0 otherwise, and continuous variables $B_{i k}$, denoting the beginning of service at $i$ by route $k$, we formally define the BITOPTW as follows:

$$
\begin{aligned}
& \min \sum_{k \in K} \sum_{(i, j) \in A} c_{i j} y_{i j k} \\
& \max \sum_{i \in V \backslash\{0, n+1\}} S_{i} z_{i}
\end{aligned}
$$

subject to:

$$
\begin{aligned}
\sum_{j \in V \backslash\{0\}} y_{0 j k} & =1 & & \forall k \in K \\
\sum_{i \in V \backslash\{n+1\}} y_{i, n+1, k} & =1 & & \forall k \in K \\
\sum_{k \in K} \sum_{j \in V \backslash\{n+1\}} y_{j i k} & =z_{i} & & \forall i \in V \backslash\{0, n+1\} \\
\sum_{j \in V \backslash\{n+1\}} y_{j i k}-\sum_{j \in V \backslash\{0\}} y_{i j k} & =0 & & \forall k \in K, i \in V \backslash\{0, n+1\} \\
\left(B_{i k}+d_{i}+t_{i j}\right) y_{i j k} & \leq B_{j k} & & \forall k \in K,(i, j) \in A \\
e_{i} \leq B_{i k} & \leq l_{i} & & \forall k \in K, i \in V \\
y_{i j k} & \in\{0,1\} & & \forall k \in K,(i, j) \in A \\
z_{i} & \in\{0,1\} & & \forall i \in V \backslash\{0, n+1\}
\end{aligned}
$$

Objective function 25) minimizes the total routing costs while objective function (26) maximizes the total collected profit. Constraints $(27)$ and $(28)$ make sure that each route starts at the defined starting point and ends at the correct ending point. Constraints $(29)$ link the binary decision variables and 30 ensure connectivity for visited nodes. Constraints (31) set the time variables and $(32)$ make sure that time windows are respected. We note that constraints (31) are not linear but they can easily be linearized using big $M$ terms.

\section{References}

Adelgren, N., Belotti, P., and Gupte, A. (2014). Efficient storage of pareto points in biobjective mixed integer programming. arXiv:1411.6538 [cs.DS].

Adelgren, N. and Gupte, A. (2016). A branch-and-bound method for multiobjective mixed integer linear programs and its implementation for biobjective problems. Working paper, Clemson University.

Aneja, Y. P. and Nair, K. P. K. (1979). Bicriteria transportation problem. Management Science, 25:73-78.

Baldacci, R., Mingozzi, A., and Roberti, R. (2012). Recent exact algorithms for solving the vehicle routing problem under capacity and time window constraints. European Journal of Operational Research, 218(1):1 - 6 .

Belotti, P., Soylu, B., and Wiecek, M. M. (2013). A branch-and-bound algorithm for biobjective mixed-integer programs. available online at http://www.optimization-online.org/DB_HTML/2013/01/3719.html.

Belotti, P., Soylu, B., and Wiecek, M. M. (2016). Fathoming rules for biobjective mixed integer linear programs: Review and extensions. Discrete Optimization, 22:341-363.

Boland, N., Charkhgard, H., and Savelsbergh, M. (2015a). A criterion space search algorithm for biobjective integer programming: The balanced box method. INFORMS Journal on Computing, 27:735-754.

Boland, N., Charkhgard, H., and Savelsbergh, M. (2015b). A criterion space search algorithm for biobjective mixed integer programming: The triangle splitting method. INFORMS Journal on Computing, 27(4):597-618. 
Braekers, K., Hartl, R. F., Parragh, S. N., and Tricoire, F. (2016). A bi-objective home care scheduling problem: Analyzing the trade-off between costs and client inconvenience. European Journal of Operational Research, 248:428-443.

Desrosiers, J. and Lübbecke, M. E. (2005). A primer in column generation. In Desaulniers, G., Desrosiers, J., and Solomon, M. M., editors, Column Generation, pages 1-32. Springer US.

Dolan, E. D. and Moré, J. J. (2002). Benchmarking optimization software with performance profiles. Mathematical Programming, 91(2):201-213.

Ehrgott, M. (2005). Multicriteria optimization, volume 2. Springer.

Ehrgott, M. and Gandibleux, X. (2006). Bound sets for biobjective combinatorial optimization problems. Computers 86 Operations Research, 34:2674-2694.

Feillet, D., Dejax, P., Gendreau, M., and Gueguen, C. (2004). An exact algorithm for the elementary shortest path problem with resource constraints: Application to some vehicle routing problems. Networks, 44(3):216-229.

Gadegaard, S., Ehrgott, M., and Nielsen, L. (2016a). Bi-objective branch-and-cut algorithms: Applications to the single source capacitated facility location problem. Working paper, Aarhus Universitet.

Gadegaard, S., Nielsen, L., and Ehrgott, M. (2016b). An instance generator for the capacitated facility location problem. github, source code (v1.0.0)

Haimes, Y., Lasdon, L., and Wismer, D. (1971). On a bicriterion formulation of the problems of integrated system identification and system optimization. IEEE Transactions on systems, man and cybernetics, 1:296-297.

Jozefowiez, N., Laporte, G., and Semet, F. (2012). A generic branch-and-cut algorithm for multiobjective optimization problems: application to the multilabel traveling salesman problem. INFORMS Journal on Computing, 24:554564 .

Leitner, M., Ljubić, I., and Sinnl, M. (2014). A computational study of exact approaches for the bi-objective prizecollecting steiner tree problem. INFORMS Journal on Computing, 27(1):118-134.

Masin, M. and Bukchin, Y. (2008). Diversity maximization approach for multiobjective optimization. Operations Research, 56(2):411-424.

Mavrotas, G. and Diakoulaki, D. (1998). A branch and bound algorithm for mixed zero-one multiple objective linear programming. European Journal of Operational Research, 107:530-541.

Raith, A., Moradi, S., Ehrgott, M., and Stiglmayr, M. (2012). Exploring bi-objective column generation. In Proceedings of the 46th Annual ORSNZ Conference, pages 288-296.

Ramos, T. R. P., Gomes, M. I., and Barbosa-Póvoa, A. P. (2014). Planning a sustainable reverse logistics system: Balancing costs with environmental and social concerns. Omega, 48:60-74.

Righini, G. and Salani, M. (2009). Decremental state space relaxation strategies and initialization heuristics for solving the orienteering problem with time windows with dynamic programming. Computers $E$ Operations Research, 36(4):1191-1203.

Sarpong, B. M., Artigues, C., and Jozefowiez, N. (2013). Column generation for bi-objective vehicle routing problems with a min-max objective. In 13th Workshop on Algorithmic Approaches for Transportation Modelling, Optimization, and Systems, ATMOS 2013, September 5, 2013, Sophia Antipolis, France, pages 137-149.

Sourd, F. and Spanjaard, O. (2008). A multi-objective branch-and-bound framework: application to the biobjective spanning tree problem. INFORMS Journal on Computing, 20:472-484.

Stidsen, T., Andersen, K. A., and Dammann, B. (2014). A branch and bound algorithm for a class of biobjective mixed integer programs. Management Science, 60(4):1009-1032.

Tam, B., Ehrgott, M., Ryan, D., and Zakeri, G. (2011). A comparison of stochastic programming and bi-objective optimisation approaches to robust airline crew scheduling. OR Spectrum, 33(1):49-75.

Tricoire, F., Graf, A., and Gutjahr, W. J. (2012). The bi-objective stochastic covering tour problem. Computers $\mathcal{E}$ OR, 39(7):1582-1592.

Vincent, T., Seipp, F., Ruzika, S., Przybylski, A., and Gandibleux, X. (2013). Multiple objective branch and bound for mixed 0-1 linear programming: Corrections and improvements for the biobjective case. Computers \& Operations Research, 40:498-509.

Visée, M., Teghem, J., Pirlot, M., and Ulungu, E. L. (1998). Two-phases method and branch and bound procedures to solve the bi-objective knapsack problem. J. of Global Optimization, 12(2):139-155. 